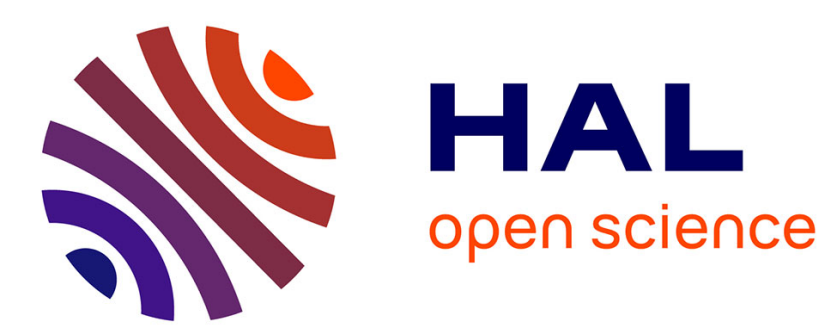

\title{
Ensemble downscaling of a regional ocean model
}

Malek Ghantous, N.K. Ayoub, Pierre de Mey-Frémaux, Vassilios Vervatis, Patrick Marsaleix

\section{To cite this version:}

Malek Ghantous, N.K. Ayoub, Pierre de Mey-Frémaux, Vassilios Vervatis, Patrick Marsaleix. Ensemble downscaling of a regional ocean model. Ocean Modelling, 2020, 145, pp.101511. 10.1016/j.ocemod.2019.101511 . hal-03052959

\section{HAL Id: hal-03052959 \\ https://hal.science/hal-03052959}

Submitted on 11 Dec 2020

HAL is a multi-disciplinary open access archive for the deposit and dissemination of scientific research documents, whether they are published or not. The documents may come from teaching and research institutions in France or abroad, or from public or private research centers.
L'archive ouverte pluridisciplinaire HAL, est destinée au dépôt et à la diffusion de documents scientifiques de niveau recherche, publiés ou non, émanant des établissements d'enseignement et de recherche français ou étrangers, des laboratoires publics ou privés. 


\title{
Ensemble downscaling of a regional ocean model*
}

\author{
Malek Ghantous ${ }^{\dagger a}$, Nadia Ayoub ${ }^{\mathrm{a}}$, Pierre De Mey-Frémaux ${ }^{\mathrm{a}}$, Vassilios \\ Vervatis $^{\mathrm{b}}$, Patrick Marsaleix ${ }^{\mathrm{c}}$, \\ ${ }^{a}$ LEGOS, Université de Toulouse, CNES, CNRS, IRD, UPS \\ ${ }^{b}$ Faculty of Physics, University of Athens \\ ${ }^{c} L A$, Université de Toulouse, CNRS
}

\begin{abstract}
We downscaled a free ensemble of a regional, parent model to a highresolution coastal, child ensemble in the Bay of Biscay. The child ensemble was forced at the open boundaries by the parent ensemble, and locally by perturbing the winds. By comparing ensembles generated by each of these forcings perturbations separately and combined we were able to consider the ensemble from either of two paradigms: (1) characterising high-resolution, coastal model errors using local and non-local forcing perturbations, or (2) downscaling regional model errors into the coastal domain. We found that most of the spread in the child ensembles was generated from the ensemble of open boundary conditions, with the local wind perturbations on their own generating substantially less ensemble spread. Together, the two sources of error increased the ensemble spread by only a small amount over the nonlocal perturabations alone. In general, the spread in sea surface height was greater in the child ensembles than in the parent ensemble, probably due to the more refined dynamics, while the spread in sea surface temperature was lower, likely due to the way the open boundary conditions were averaged. Deep below the surface, though, the child ensemble featured a large spread even where the parent model's spread was very weak. This enhanced error response is a promising result for an ensemble data assimilation system, as it could be exploited to correct the model deep below the surface.
\end{abstract}

\footnotetext{
*Manuscript accepted for publication in Ocean Modelling, doi: https://doi.org/10.1016/j.ocemod.2019.101511. This preprint is licensed under the Creative Commons Attribution-NonCommercial-NoDerivatives 4.0 International License. To view a copy of this license, visit http://creativecommons.org/licenses/by-nc-nd/4.0/.

${ }^{\dagger}$ Now at Météo France, Toulouse; Corresponding author
} 
Keywords: Ensemble modelling, downscaling, model uncertainties, coastal modelling, Bay of Biscay, data assimilation

\section{Introduction}

We downscale geophysical models so that we can increase the resolution in a region of interest, without incurring the computational cost and the difficulty of increasing the resolution everywhere in the model domain. This modest aim leads to anything but a straightforward problem to solve, though, for the difficulties associated with nesting models are manifold, and the highresolution child model cannot simply be regarded as a more detailed version of the parent model. A corollary of this is that the errors in the parent model cannot be expected to translate in a straightforward way into errors in the child model. It is this problem - the downscaling of model errors - which we concern ourselves with here, specifically in ocean circulation models.

The promise of higher resolution models to provide more detailed descriptions of the ocean balances against some inconvenient physics at small scales. As Sandery and Sakov (2017) show, while increased detail is possible, and useful, this does not necessarily mean increased accuracy, with the higher complexity of the small-scale flow even deteriorating the large-scale flow through an inverse energy cascade. There are further problems to address with the open boundary problem. Quite apart from the difficulty of matching solutions of different spatial and temporal scales, the internal variability of the downscaled model is decoupled from that of the parent model, which can lead to divergent solutions - an undesirable trait when the parent model outperforms the child. Sometimes this divergence can be redressed, as Katavouta and Thompson (2016) showed by applying a spectral nudging technique in the deeper regions. This maintained the parent model's accuracy there, while giving free reign to the child model in the coastal region, where the parent model was unable to resolve many of the processes. With all this in mind, it would be fruitful then to explore the behaviour of errors in a downscaled model system.

In this study, we attempt to characterise a downscaled coastal model's errors from ensembles of perturbed simulations. We do this principally by examining the ensemble's statistics - above all the ensemble variance or, equivalently, its standard deviation, which is often referred to as the ensemble's spread. There are many sources of error in a model, due to deficiencies in 
the physical model, the numerical scheme and discretisation, and errors in the initial and boundary conditions and the forcing fields. By narrowing our focus onto a subspace of the full model error space, we can better understand the sensitivity of the model to a specific set of error sources. For us, this subset consists only of surface winds and the open boundary conditions. Understanding the model error subspace will guide us in future efforts in assimilating data, where corrections to the model will only be possible for variables sensitive to these two sources.

Experimenting with forcing error perturbations in ocean models is not new, of course, even for nested models. To name but a few studies, Echevin et al. (2000) perturbed the initial conditions while leaving all other forcing variables untouched; Auclair et al. (2003) examined the effect on a coastal model of perturbing, in turn, the initial density field, a prominent slope current in the domain, the wind field and river discharges; Jordà and De Mey (2010) perturbed the wind and the initial, analytic velocity profile; Kim et al. (2011) perturbed the winds, the initial conditions and the open boundary conditions, the last of these by calculating EOFs for the boundary conditions and, with random amplitudes, superimposing a linear combination of EOF modes onto them (in fact, the same basic procedure we and others have used to perturb the wind). The distinguishing feature of the work we are presenting is thus the use of an ensemble of open boundary conditions. By defining our open boundary perturbations from a pre-existing parent model ensemble, we achieve something similar to Kim et al. (2011) as far as the coastal model error space is concerned, but we gain the ability to compare our coastal model errors with the parent model errors - in particular, our coastal model's error space may be thought of as representing a refinement of the parent model's error space. At this point, the problem may be viewed from two equivalent perspectives: one is that of characterising coastal model errors by generating a coastal model ensemble, and the other is that of downscaling errors from a larger-scale (parent) model ensemble. Though philosophically different, functionally these two paradigms amount to the same thing, as long as the former is approached like the latter, using an ensemble of open boundary conditions drawn from the parent model ensemble.

Since the parent ensemble was generated by perturbing solely the wind, and we further perturb the local winds in the coastal ensemble, it is evident that the only forcing variable directly perturbed is that of the wind. Thus the open boundary conditions provided by the parent ensemble can be considered to be a conduit through which the effects of non-local wind perturbations can 
enter the coastal domain. It may seem limiting to use only one error source to generate our coastal ensemble, but the implicit assumption underlying this choice - that the model is perfect and that all the errors come from the wind forcing - has the advantage that it enables us to concentrate on understanding the consequences of one source of error. And indeed the wind turns out to be a good choice, as Vervatis et al. (in review, 2019) found that perturbing the wind in a regional model (the very same regional model configuration as the parent model used in this study) had a much greater effect than the other physical and biogeochemical perturbations they tested. Being restricted to wind perturbations also allow us to compare like with like when comparing the parent and child ensembles. To that end, we follow in the footsteps of other studies where, in those too, only the wind was perturbed (for example those by Le Hénaff et al. (2009) and Kourafalou et al. (2015)).

There are several ways one might choose to categorise the ensemble error behaviour, the most obvious being with gross quantities like calculating the mean ensemble spread over time. But it is informative to look beyond bulk quantities and see where, as well as by how much, the spread changes. To address this we subdivide the domain into deep-water, continental shelf and intermediate zones. We also generate surface and cross-section maps to see what the error pattern looks like at a given point in time and space, both at the surface and below the surface. Different variables should be expected to behave differently, too, and this should also depend on the regime; near the river mouth we should see strong errors in salinity and temperature, but sea surface height ought to vary most in deep water where the large eddies dominate in the signal. We therefore consider these variables separately and compare them between the parent and child ensembles.

After a brief and general description of the Bay of Biscay (section 2), and of the regional parent and coastal child models (section 3), we discuss in some detail how the wind perturbations were calculated and how we used the open boundary ensemble to generate our ensembles (section 4). The bulk of the paper is given over to an exploration of these ensembles (section 5). We treat the surface extensively before diving beneath, showing how surface wind perturbations can lead to large errors in specific zones under the surface, and how this is also model-dependent. Because of its highly non-isotropic nature, the behaviour of the river plume when subjected to wind perturbations holds its own interest, so we treat this separately (section 5.5) before concluding with a general discussion. 


\section{The Bay of Biscay}

We shall present only a very brief summary of the major physical processes in the Bay of Biscay, and in particular in the small domain at the heart of this study. We invite the reader to consult the cited references for more detail.

The Bay of Biscay is really a gulf situated in the eastern Atlantic Ocean, with its land boundaries formed by Spain's northern coast and France's western coast. The Bay of Biscay and its bathymetry are shown in figure 1, which also shows the domain boundaries for the regional parent model used in our experiments, and the smaller coastal domain. The continental shelf is clearly visible in the figure. It can be as narrow as about $20 \mathrm{~km}$ on Spain's northern coast near Bilbao, but it broadens greatly as one travels north along the French coast, and less so westward along the Spanish coast. For our small coastal domain, shown in figure 2, the important submarine features are the Gouf de Capbreton, a striking submarine canyon, and the Plateau des Landes, which is really a gentle slope descending from the shelf into the abyssal plain. Although the only river directly modelled in our domain is the Adour, which meets the sea near Bayonne, the Gironde's plume enters from the domain's northern boundary and is a major source of fresh, cool water along the coast. The discharge from both rivers is significantly cooler than the water in the Bay, so the river plumes can be identified from the sea surface temperature maps, a fact we exploit in this study.

Even though our model domain covers only a small corner of the Bay of Biscay, an array of different physical processes may be found therein. There are the river plumes of cooler, fresh water, which are an important part of the dynamics over the shelf; the river discharge is greatest during the winter months (Puillat et al., 2006). An intermittent along-shore current, the Iberian Poleward Current, extends along the Spanish coast in late Autumn and Winter, and occasionally follows the slope as it turns northward (Rubio et al., 2013; Kersalé et al., 2016). This current is also associated with mesoscale, anticyclonic slope-water eddies (SWODDIES), which are provoked by bathymetric features such as undersea canyons, which interrupt the current's flow and send jets of slope water into deep water regions (Pingree and Le Cann, 1992).

Of particular interest for this study, the Plateau des Landes plays host to some intense mesoscale activity, with cyclones and anti-cyclones of between 50 and $80 \mathrm{~km}$ diameter (Le Cann and Serpette, 2009; Solabarrieta et al., 
2014). Beneath the surface, incursion of water masses such as the Mediterranean Intermediate Water can lead to strong and deep sub-surface gradients in temperature and salinity (van Aken, 2000).

The barotropic tidal amplitudes in the Bay of Biscay can be extremely large, over $1.5 \mathrm{~m}$ in some places over the shelf, dominated by the semi-diurnal lunar tide (M2) (Le Cann, 1990). As the tidal current crosses the shelf break, quite complex baroclinic flows and internal tides can be generated, some of whose energy dissipates by adding to the vertical mixing (Pairaud et al., 2010). In our model domain, because the shelf is narrower, the tidal currents are not as strong as they are further north (Le Cann, 1990; Toublanc et al., 2018).

\section{The ocean circulation models and their configurations}

Before we discuss ensemble generation, we shall say a few words about the models themselves. We give only brief descriptions of them here as they have been more thoroughly described elsewhere. We refer to the highresolution coastal model as the "child", or coastal, model and the regional model supplying the open boundary conditions as the "parent".

The parent ensemble is the free ensemble produced by Vervatis et al. (2016) and described fully in their paper. It was generated with the NEMO model (Madec and the NEMO team, 2008) on a $1 / 36^{\circ}$ grid on a domain encompassing the Bay of Biscay and the western part of the English Channel. This configuration, named BISCAY36, was based on that of Maraldi et al. (2013).

The coastal model is Symphonie, which is described in a series of papers by Marsaleix et al. (2006, 2008, 2009a, 2011, 2012); Estournel et al. (2005, 2009) and Pairaud et al. (2008). Over the last decade, the Symphonie model has been used in several different configurations to model the Bay of Biscay: see the studies of Pairaud et al. (2008) and Pairaud et al. (2010) for the barotropic and internal tides; Le Hénaff et al. (2009) for the general circulation; Herbert et al. (2011) on the Iberian Poleward Current along the northern Spanish coasts; Herbert (2012) for the surface circulation response to storm Klaus in January 2009 and, recently, Toublanc et al. (2018) for the impact of tides on the open-boundary conditions. These studies have shown that Symphonie was able to simulate the main circulation patterns in the Bay of Biscay from daily to seasonal time scales. 
The domain, whose limits can be seen in the bathymetry plots in figure 2, is much smaller than the parent model's - it forms a box over the southeastern corner of the Bay of Biscay extending northward up to just beneath Arcachon on France's southwest coast, and westward ending a little before Santander on Spain's northern coast (figure 1). The resolution is set to $500 \mathrm{~m}$ between grid points in both horizontal directions, a substantial improvement over the parent model's roughly $2.5 \mathrm{~km}$ resolution. This enables it to better resolve mesoscale eddies - perhaps even allowing the resolution of sub-mesoscale eddies - fronts and river plumes. The model bathymetries are different, too: for the coastal model we used a new, highresolution bathymetry (Lyard, 2015), shown in figure 2. While the parent model defines depth with $z$ values, the coastal model uses $\sigma$ layers. We refer to the specific configuration of the Symphonie model in this high-resolution coastal domain as BOBLAND.

The meteorological fields for both model configurations are the 3-hourly operational analyses from the European Centre for Medium-Range Weather Forecasts' (ECMWF) high-resolution model, and are thus identical for the parent's and child's unperturbed runs.

The child model is not two-way nested in the parent model, but rather uses an independent run of the parent model to define its open boundary conditions (that is, "offline"). The method for handling the open boundaries is clearly of consequence for this study, but we defer this to the discussion on downscaling in section 4.2.2.

A summary of some of the features and configuration parameters for both models is given in table 1 .

In this paper we perform sensitivity studies, so we have not sought to verify in detail the realism of the model beyond what these prior studies have done. We nevertheless thought it prudent to check that our results were in line with observations. Unfortunately, very few observations were available to us for our period of study in our domain. We have used the L3S daily satellite SST fields (Orain, 2016) but the coverage is very poor due to the frequently cloudy conditions in the Bay of Biscay in winter. Comparisons of the unperturbed runs for both the parent and child with those days with good coverage showed few differences of more than half a degree Celsius. We have also checked that our results are qualitatively consistent with the information found in the literature. For instance, the observations of SST and HF radar presented by Solabarrieta et al. (2014) show a large eddy similar to the one that persists throughout the study period. While the size 
and location of the eddy are consistent between the coastal model and the data, the parent model's eddy properties seem less realistic. Also, we found that the SST gradient over the eastern shelf and at the edge of the Adour's plume was in good agreement with Solabarrieta et al.'s 2014 observations; along the Spanish shelf it was however too warm, maybe because of a local recirculation (not shown).

\section{Ensemble generation}

One of our main assumptions is that the model errors result principally from errors in the forcing variables. If we know the distribution of these errors, or we can guess at it, we can generate an ensemble of perturbed forcing variables which follow it. If we then apply this ensemble of perturbed forcings to the model we can generate an ensemble of model realisations, each member corresponding to a particular forcing perturbation. This model ensemble can then provide a useful estimate of the model error covariances. Strictly speaking, of course, the estimate is only of the subspace of error covariances related to the forcing variables we perturb, but if these forcing variables dominate the others then this is adequate. In this study, where our focus is on downscaling an ocean circulation model, we only directly perturb the winds and the open boundary conditions, although these latter are themselves generated from perturbations to the parent model's winds. Vervatis et al. (in review, 2019) found that the wind was, and by quite some way, the most important source of error among those that they tested.

We describe in turn the procedure for perturbing the wind locally and how we utilised the ensemble of open boundary conditions.

\subsection{The wind perturbations}

We followed Auclair et al. (2003) and Le Hénaff et al.'s 2009 method for perturbing the wind field, which is also the method used by Vervatis et al. (2016). Our basic assumption is that the uncertainties in the ECMWF wind fields have the same spatial and temporal structure as the wind variability. Consequently, the perturbations are built from the empirical orthogonal functions (EOFs) of the ECMWF wind fields in the BOBLAND domain over the period desired - for this study, this is December 2011 to March 2012.

Let $\mathbf{U}$ be the matrix containing both zonal and meridional wind velocities. It may be decomposed into its temporal mean and an anomaly matrix,

$$
\mathbf{U}=\overline{\mathbf{U}}+\mathbf{U}^{\prime}
$$


Now for a matrix $\mathbf{U}^{\prime}$ containing both zonal and meridional wind anomalies, and of dimension $k \times l$ where $k$ and $l$ are the temporal and spatial dimensions respectively, we may perform a singular value decomposition giving us the expression

$$
\mathbf{U}^{\prime}=\mathbf{X} \boldsymbol{\Lambda} \mathbf{Y}^{\mathrm{T}},
$$

where $\mathbf{X} \in \mathbb{R}^{k \times j}$ and $\mathbf{Y} \in \mathbb{R}^{l \times j}$ are composed of orthogonal vectors and $\Lambda \in \mathbb{R}^{j \times j}$ is diagonal, whose non-zero elements $\sigma_{j}$ are the singular values of $\mathbf{U}^{\prime}$. The dimension $j$ corresponds to the number of modes, of which only the first few are computed, using the method described by Toumazou and Cretaux (2001). To decide how many to keep, we can use the singular values from $\Lambda$ to determine what percentage $P_{j}$ of the total variance each mode $j$ represents:

$$
P_{j}=\frac{\sigma_{j}^{2}}{\operatorname{Tr} \Lambda^{2}} .
$$

where Tr indicates that the trace of the matrix is taken. For our EOFs the first two modes explained $\sim 64 \%$ and $\sim 29 \%$ of the variance respectively, and there was a steep drop to the third mode at only a little more than $2 \%$, after which the percentage for subsequent modes fell gradually. Figure 3 shows the temporal standard deviation of the first five modes, where the dominance of the first zonal and second meridional modes is made clear, as is the much weaker contribution from higher-order modes. We therefore decided to keep only these first two modes, together explaining about $93 \%$ of the total variance.

The EOF modes may be recombined with random numbers $\epsilon_{i, j}$, one for each ensemble member $i$ and each spatial mode $j$, to produce a wind perturbation which is added to the original wind field. Following Lucas et al. (2008), we choose the random numbers from a Gaussian distribution, in pairs of positive and negative numbers of the same absolute value. The probability density function is then

$$
p(\epsilon)=\frac{1}{\sqrt{2 \pi s^{2}}} e^{-\frac{\epsilon^{2}}{2 s^{2}}},
$$

where $s$ is the standard deviation. The perturbation can be expressed as

$$
\widetilde{\mathbf{U}}_{i}=\sum_{j=1}^{2} \epsilon_{i, j} \mathbf{x}_{j} \sigma_{j} \mathbf{y}_{j}^{\mathrm{T}},
$$


and the wind field for ensemble member $i$ is then given by

$$
\mathbf{U}_{i}=\mathbf{U}+\widetilde{\mathbf{U}}_{i} .
$$

If our ocean model were linear, the pairs of Gaussian random numbers would guarantee that the ensemble of simultions be Gaussian with zero mean. Following Vervatis et al. (2016), we assume an error standard deviation of $s=0.3$ for equation (4), reflecting a presumed observational error of $30 \%$ for the wind. (It's worth mentioning that Le Hénaff et al. (2009) assumed an error of 0.2 , but chose the value of 0.3 to deliberately inflate the ensemble dispersion. Since there are many other sources of error we have not perturbed, and noting that our ensembles are not especially long either, we feel that the choice is reasonable for our configuration, whatever the real error value.)

To prevent the wind perturbations from being correlated between members, the random numbers must be replaced by a new set generated with the same Gaussian and pairing criteria, and uncorrelated with previous sets. All the same, the rate at which we change them should not be too high, otherwise coherent weather patterns would not have enough time to develop. Vervatis et al. (2016) and Le Hénaff et al. (2009) chose to update the random numbers every five days. Their choice also corresponded to the decorrelation time of the wind EOFs. Although their EOFs are on a much larger spatial scale than ours, ultimately it is still an arbitrary decision, so we did not seek to replicate this methodology for our reduced domain, preferring instead to follow their example and use five days as well. To avoid shocks when updating from one set of random numbers to the next we linearly interpolated them in time, ensuring a smooth evolution from one perturbation to the next for all times.

\subsection{An ensemble of open boundary conditions \\ 4.2.1. Generating the parent ensemble}

Downscaling a regional model to a high resolution coastal model implies using the regional, parent model to prescribe the open boundary conditions for the coastal, child model. In our study we want to take this a step further: we want to downscale a regional model ensemble, so for each member of our coastal ensemble we use a different member of the parent ensemble to force the open boundaries. From the point of view of the coastal ensemble, this is equivalent to saying that its open boundaries are being perturbed. Other ways of perturbing them could be envisaged, such as the methods tested by 
Jordà and De Mey (2010), but by using the parent ensemble we maintain the dual paradigm of ensemble downscaling and - equivalently - perturbation of the open boundary conditions. For the case where we perturb the winds locally as well, this adds extra degrees of freedom to our ensemble.

Our parent ensemble is the regional free ensemble described by Vervatis et al. (2016), generated, as we have said, by perturbing the ECMWF winds using the same EOF method we have just presented. However, their EOFs were calculated over the whole BISCAY36 domain, for a different period from ours and from daily averages of the wind fields as opposed to the 3-hourly fields we used. The temporal standard deviations of the first five of their EOF modes for the BISCAY36 configuration are shown in figure 4, where the view has been restricted to the BOBLAND domain. If we compare them to the EOFs we calculated for the BOBLAND configuration, shown in figure 3, we see that although both EOFs are dominated by the first zonal mode, thereafter they are markedly dissimilar. The BOBLAND EOFs were calculated only over the BOBLAND domain, so we see a slightly more complex structure, and the second meridional mode is stronger relative to its neighbouring modes - first meridional and second zonal - than in BISCAY36. Vervatis et al. (2016) kept the first six EOF modes to generate their wind perturbations, but even by only keeping the first two modes in BOBLAND we might expect stronger and more varied wind perturbations, both because of the stronger first zonal and second meridional modes, and because of the more structured second zonal and first meridional modes. We test this hypothesis in section 5.3.3.

There are two other differences between the way we and Vervatis et al. (2016) handled the time-dependent component of the perturbations. Firstly, they didn't use every index from the EOF time series like we did, but instead every fifth one, interpolating them the same way as the random numbers. Secondly, when we started the ensemble runs we set the first random number to zero for all members, so that the first perturbation would ramp up from zero. The omission of this step created a spike in the parent ensemble's spread (discussed in section 5.3.1).

\subsubsection{The downscaling method}

Some thought was needed about the manner in and the extent to which the regional model should influence the coastal model. The regional model's fields were provided as daily averages, where the mean was taken over 25 instantaneous fields spaced an hour apart. The fields used to force the open boundaries of the child model are temperature, velocity, and sea surface 
height. The "25-hour" averaging for the parent ensemble's fields does a fairly good job of removing the tides from the sea surface height and velocity fields, but some signals are still present, principally an artefact due to aliasing. This artefact has a period of around 14 to 15 days, with an amplitude of about a couple of centimetres in deeper water. It acts as a forcing on the coastal model, producing likewise a signal with a fortnightly oscillation of up to about $4 \mathrm{~cm}$ over the shelf or $2 \mathrm{~cm}$ in deep water. A more thorough discussion of the problems associated with the way these open boundary conditions have been averaged was presented by Toublanc et al. (2018), though the context is that of a much larger scale coastal model.

The boundary condition in the Symphonie model is of the radiative Flather type. (For a complete description on the way Symphonie handles its open boundary conditions, we refer the reader to Marsaleix et al. (2006) and Marsaleix et al. (2009b).) We use a nudging layer of 30 grid points for the main experiments, corresponding to $15 \mathrm{~km}$ in the BOBLAND configuration. At the boundary the nudging time scales are at $\tau=0.1$ days for the barotropic velocity and $\tau=1$ day for the baroclinic velocity. Marchesiello et al. (2001) applied a cosine half-period to reduce these values to zero, which is the method Symphonie uses, to wit,

$$
\tau_{r}(d)= \begin{cases}\frac{\tau}{2}\left(1-\cos \pi \frac{s_{L}-d}{s_{L}}\right), & \text { for } d \leq s_{L} \\ 0, & \text { for } d>s_{L}\end{cases}
$$

where $\tau_{r}$ is the reduced nudging time scale at distance $d$ from the domain boundary, for a nudging layer of width $s_{L}$.

We also considered using a narrower nudging layer of only 10 grid points before settling on 30. Comparing unperturbed model runs of both nudging layer widths with sea surface temperature data, we were unable to discern much difference between the two. However, a wider nudging layer could serve to constrain the coastal ensemble spread: for example if the nudging layer is too wide, the boundary conditions will impose themselves to the detriment of any other perturbed dynamics. On the other hand, a wider nudging layer, we reasoned, should cause the open boundary conditions to have more influence further into the domain. For our application this may have benefits, since it means, all other things being equal, that the coastal model ensemble will capture more of the parent ensemble's spread. This was desirable too in subsequent work, where we we wished to preserve the large scale circulation 
of the parent model while assimilating data at the same time (to be presented in a future paper). Thus the wider nudging layer was preferred, although in the sections that follow we discuss the impact of the nudging layer width on the ensemble where pertinent.

\section{Ensemble behaviour and statistics}

\subsection{Introducing the ensembles}

We generated several ensembles in the BOBLAND configuration, whose defining characteristics are summarised in table 2. Each one consists of 50 members, from which the unperturbed run is excluded. Of these ensembles, we concentrate on three, plus the parent ensemble, with the others used mostly to answer specific questions raised by the first three and to test some of our assumptions. The four principal ensembles on which the bulk of our analysis is based are the parent, WIND, OBC (Open Boundary Condition) and WIND $\times$ OBC ensembles, the names corresponding to the forcing being perturbed. For WIND $\times$ OBC the two types of perturbations are applied at the same time. When we perturb only the wind we use the same unperturbed open boundary conditions for all members, and likewise when we perturb only the open boundary conditions we use the same unperturbed winds for all members. The coastal ensemble runs begin after a model spinup, starting on September 12, 2011 and ending on November 30. At this date all of the ensemble members are identical and have the exact same open boundary conditions and winds as the unperturbed run, and they diverge from then on.

In what follows, all ensemble statistics were computed over the 50-member ensemble of perturbed runs (excluding the unperturbed member). To better represent and compare the ensemble statistics of each ensemble proper, without contamination from the parent ensemble in the nudging layer, the spatial averages exclude points which would fall inside the 30-point nudging layer of the BOBLAND domain. Similarly, to compare our ensembles with the parent ensemble, statistics for the parent were only computed over the BOBLAND domain, again excluding points which fall inside the nudging layer.

The statistical quantity which primarily interests us is the ensemble variance, or equivalently the ensemble standard deviation (which is also referred to in this paper and in the literature as the ensemble spread). This tells us to what extent the perturbations have led the ensemble to diverge, and may be interpreted as a measure of the error in the model resulting from 
an imperfectly known forcing. (This is of particular significance in a data assimilation context: we may think of it of as indicative of how amenable the model is to correction by data assimilation, since the more error there is in the model then the more weight the observations will carry in the analysis for any given observational error.)

We follow Vervatis et al. (2016) in treating the first month of the ensembles (i.e. December) as an "ensemble spin-up" period, where the ensemble members have still not diverged enough to give useful statistics. We hence exclude the first month of our ensembles from the temporal moments we examine later in this paper, but it can still be useful to look in here: one reason is that it shows how the ensemble spread grows as the model perturbations take effect. But there are also some dynamics which only, or mostly, occurred in this period.

\subsection{Quantifying the interaction with tides}

Exploring the model error subspace will guide us in future choices for data assimilation experiments. In particular, when dealing with SSH observations (such as altimetric data), the question arises whether the tidal signal should be assimilated together with the residual circulation. We therefore need to understand if the tidal dynamics are represented in the model error subspace. Indeed, tides influence and are influenced by the circulation, so perturbations on wind and open boundary conditions could in principle perturb tides. Here we seek to quantify the effect of the perturbations on the tidal elevation signals. To do this we need to remove the tidal signal from the elevation. For accuracy we run a harmonic analysis on the surface elevation, which requires a time series with a high sampling rate. The analyses are therefore done at a few representative locations where we recorded these high-frequency time series with a sampling rate of $23^{\prime} 15.5^{\prime \prime}$; they are marked in figure 2 .

We can estimate the effect of the perturbations on the tides by calculating the ratio of the ensemble spread of the tidal signal, to the ensemble spread of the residual circulation. For elevation $\eta$, this may be expressed as

$$
\frac{\operatorname{std}\left(\eta-\eta^{\prime}\right)}{\operatorname{std}\left(\eta^{\prime}\right)}
$$

where the prime indicates detiding by harmonic analysis. Figure 5 shows this ratio for each of the ten locations marked in figure 2 . The highest value is about 0.16 for a point on the shelf, but most of the time the values 
are closer to 0.05 , only breaking the 0.1 threshold on a few occasions. For points over deep water, the ratios are even smaller. One sixth is not a completely insignificant contribution to the ensemble spread, but it is an absolute maximum, and other high values only occur occasionally and over the shelf. Consequently, we consider that the perturbations' effect on tidal elevations is weak enough when compared with the residual circulation that it can be neglected. Thus in the following sections we consider only the modification of the residual circulation, using detided daily fields of SSH, where the detiding is done online by subtracting the tidal forcing from the model output fields before computing the daily average.

\subsection{Ensemble spread of surface variables}

Since it is at the surface that our forcing perturbations are generated (bearing in mind that the parent ensemble was generated by wind perturbations too), so it is that we begin our examination of the ensembles at the surface too.

\subsubsection{Time series of the spatial mean spread}

Figures 6 and 7 show time series for the spatial mean of the ensemble spread of sea surface height (SSH) and sea surface temperature (SST) respectively. We shall focus initially on the top-left plot in these figures, in which the spatial means are taken across the whole of the BOBLAND domain. For the moment we discuss only the four principal ensembles: the WIND, OBC, WIND $\times$ OBC and parent ensembles, all drawn in black.

We first address the most prominent feature of the SSH curves. Except for the WIND ensemble, there is an extremely high spike in the spread at the beginning of the time series. This is related to the manner in which the parent model's wind perturbations were abruptly introduced. The subsequent spikes are, on the contrary, not artefacts but are related to a strong, synchronous sea surface height change from the parent model, which is accentuated on the shelf. The SSH curves for the WIND ensemble show no such spikes as this was the only ensemble not to use the parent ensemble for its open boundaries. Because we treat this first month as a spin-up, the anomalous initial spike is excluded from any temporal statistical calculations we make.

If we examine the full time series, we see that the OBC and WIND $\times$ OBC ensembles have a greater overall spread in SSH than the parent ensemble, a trend which is largely inverted for the SST. Meanwhile, the WIND ensemble 
almost always has the least spread of all the ensembles, except for SST during the ensemble spin-up phase. This is because so much of the SST spread is generated by the cold river plume over the shelf, and during the ensemble spin-up the parent ensemble's perturbations have not had the time to propagate from the open boundaries to the near-coast, leaving the interior initially unaffected unless the coastal ensemble's winds are perturbed. In a similar fashion, the parent ensemble's spread is initially lower than the WIND ensemble's until non-local perturbations have had time to reach the shelf.

That the SST and SSH should respond differently to the forcing perturbations is not in itself remarkable (we discuss it further below), however it may seem surprising at first that the parent ensemble, in which only the winds are perturbed, should have a greater spread in SST than the higher-resolution WIND $\times$ OBC ensemble, where the perturbations from the boundary are superposed on the wind perturbations. There are several mechanisms that might contribute to this. Firstly, the high-resolution coastal model is able to produce a more complex velocity field, with more coherent eddies at smaller scales. Even if the SSH fields are more distinct from member to member, this could result in more horizontal diffusion of scalars like temperature, leading the ensemble members to look more alike in those variables. But there is more to it than that, because it also depends on how sub-grid scale diffusion is handled in each model. Even though the tracer diffusion scheme is the same for both models, the momentum diffusion is different, and the numerical diffusion, resolution, time step and other features, which are specific to each model, will influence horizontal diffusion. Because the differences between the models run so deep, a thorough comparison is impractical. A simple test would be to see the effect of increasing horizontal diffusion of tracers in the coastal model alone, which we did by doubling it. The result partly confirmed our hypothesis about diffusion and spread, in that the ensemble spread decreased as horizontal diffusion increased. However, increasing the horizontal diffusion parameter also lead to more diffusion of the velocity field, in turn reducing the SSH spread. This implies that scalar diffusion cannot be the sole mechanism responsible for the inverted spread behaviour.

To further get a sense of the relationship between the complexity of the eddy field and horizontal diffusion, we looked at time series of spatial standard deviations for scalars and SSH. Calculating the temporal correlation coefficients of these time series for the unperturbed member of both parent and child model configurations was inconclusive, however: they do turn out to be negative, but they are very weak, with absolute values less than 0.3 (ex- 
cept for SST with SSH for the parent model, whose value is -0.59). Despite the inconclusiveness of these findings, and the equivocal role that diffusion appears to play in the different behaviour of the parent and child, in the following section we examine evidence suggesting that indeed the parent model diffuses tracers rather less quickly than the coastal model.

The second reason for the inverted response of the SSH and SST spreads in the two models has to do with the way we prescribe the open boundary conditions themselves, which is as daily means. These are interpolated by the coastal model for the current time step, but higher frequency information is irretrievably lost. The coastal ensemble is thus being constrained into a lower frequency error space than the parent ensemble by means of the lower frequency forcing at the open boundaries. This latter explanation leads to a contradiction, for the loss of high frequency information should lead to a reduction in spread of all variables, yet it doesn't. We refer back to the first reason to explain this discrepancy: the coastal model's higher resolution and therefore greater ability to resolve eddies. The more defined eddies are stronger and more localised in space, so their displacement must impose a more marked difference between ensemble members than the weakly resolved eddies of the regional model. This justifies the ensemble downscaling paradigm, since despite the mechanism by which we may expect the ensemble spread to be reduced - the loss of information at the open boundaries - the spread of SSH nonetheless increases.

Finally, a big contributor to the reversed trend of SSH and SST spreads appears to be directly attributable to the Adour's plume, which we consider in the next section.

\subsubsection{Subdividing the domain}

In a previous ensemble study at the basin scale with the Symphonie model, Kourafalou et al. (2015) emphasised the existence of distinct regimes of model error over the shelf and in deep water. To find out how these regimes differed in our ensembles we divided the BOBLAND domain into three subdomains according to depth. The subdivisions are as follows: deep water, which we define as more than a thousand metres depth, and which encompasses the abyssal plain and much of the gently sloping Landes Plateau (top right plots in the figures); an intermediate region where depths vary between a thousand metres and 150 metres, which we may associate with the continental slope (bottom left plots); and the continental shelf with depths up to 150 metres (bottom right plots). These regions are defined based on the 
smoothed bathymetries seen by the respective models, which although not precisely the same as each other are close enough (as illustrated in figure 2). Time series of the spatial means of ensemble spread for each subdivision are shown in the remaining plots in figures 6 and 7.

The SST spread is greatest over the shallow waters of the shelf, where the river plumes are still coherent and can create very big and sharp differences in SST. By contrast, the SSH spread is smaller here, and greater in deep water, where large, spatially coherent eddies and steric effects from a deeper water column have more influence. But there are some peaks in SSH spread over the shelf for all ensembles except for the WIND ensemble. These peaks are driven by wind-related processes in the parent model, and are discussed by Vervatis et al. (2016). They manifest as large, synchronous increases in the SSH everywhere, but especially over the shelf.

The SSH spread over the slope is most of the time somewhere in between the shelf's and the deep water's. The SST shows something different, though. From mid-February, the spread of all the ensembles is lower here than over either the shelf or the abyssal plain. Meanwhile, at the end of December, and from mid-January through to mid-February, the parent ensemble's spread is far greater over the slope than that of the coastal ensembles. The great difference between the ensembles may be explained by looking at the surface maps in figure 8 , where we see that the parent model produces a more spatially coherent river plume, and during those periods of large spread in the parent ensemble it extends out some distance from the coast, being entrained by the deep-water eddies. The coastal model diffuses the plume earlier, so it does not extend as far out. Naturally, the further out the plume remains undiffused, the more it can be deviated by the circulation, and the higher the overall spread will be there than if it stayed near the coast and diffused quickly. From mid-February, the plume doesn't extend as far out, with the cooler river water tending to hug the coast, where the spread remains quite large. The mesoscale eddies are the other major source of ensemble spread at the surface, but, as is hinted in the SST maps and becomes clear when looking at the $\mathrm{SSH}$, these circulate mainly in deep water and do not greatly influence what is happening over the slope. The slope is, in a sense, situated in between two highly active regimes, insofar as SST spread goes.

This leaves us again to consider the greater spread in SST of the parent ensemble compared with the child ensembles. Looking again at the surface maps for SST and SSH in figure 8, we can see that coinciding with the large eddy's centre (high SSH) are higher temperatures. These temperatures are 
not only higher for the parent model, but the gradient is also steeper; in other words, while the parent model's eddy has a less distinct signature in SSH than the coastal model, it has a conversely more distinct signature in SST. This distinct signature naturally leads to a higher spread in SST for the parent ensemble, and once more suggests that the parent model diffuses tracers more weakly than the coastal model.

We also produced plots for the sea surface salinity (SSS) but they do not add much to the argument, so we do not present them. The two salient points are these: the pattern of the SSS spread follows quite closely that of the SST, including the enhanced spread in the parent model over the slope; and the spread of the SSS is reduced by an order of magnitude in deep water. The former observation is easily explained since both tracers are controlled by fairly similar mechanisms: the river plumes on the shelf and the eddies over the abyssal plain. The latter is simply because, unlike the temperature, the biggest source for changes in salinity is the fresh water discharge from rivers, which is most pronounced near the coast.

\subsubsection{Experimenting with the wind perturbation}

The manner in which we perturbed the winds involved some choices based more on convention than on physical law. To determine how much these choices influenced the results we generated a few extra ensembles, described in table 2. Note that for want of computer resources these experiments were only performed for part of the period under examination, some not exceeding the ensemble spin-up period. The spatial means of SST and SSH spread for these ensembles are shown in figures 6 and 7 along with the four principal ensembles.

It should be expected that increasing the wind perturbations should lead to greater ensemble spread in response. The ensemble s05 bears out this intuitive hypothesis. It is the exact same configuration as for our WIND ensemble, with the same distribution of random numbers $\epsilon_{i, j}$ used to construct the wind perturbations (equations (4) and (5)), except with a higher error standard deviation of $s=0.5$, instead of 0.3. The conclusion holds true for both the SSH and SST and all subdivisions of the domain, although near the end of the ensemble spin-up period the difference is sometimes very small.

In section 4.2, we discussed how our EOFs were calculated over a smaller domain and a shorter time period than those of Vervatis et al. (2016), and that we kept only the first two modes. The smaller domain could serve to increase the structural detail for the same number of modes by virtue of 
excluding contributions from outside the domain, but the shorter time frame could have the opposite effect because it doesn't capture long-term changes; similarly, restricting ourselves to only two modes precludes some of the smallscale structure. The two sets of EOFs are, in short, not directly comparable. This prompted two questions: firstly, what do we lose by only keeping the first two modes, and secondly, what if we were to use exactly the same wind perturbations as those which generated the parent ensemble? For the second question, one intriguing possibility is that for any particular member, the open boundary conditions would set up an ocean circulation pattern in the child model which could be reinforced by matching winds - a kind of resonance effect which would maintain a larger spread. Or, looked at another way, if we used different wind perturbations than those used to generate the parent ensemble, those perturbations might dampen the differences generated by the ensemble of open boundary conditions.

To answer the first question we generated the EOF10 ensemble, which is the same as our WIND ensemble except that we kept the first ten EOF modes instead of only the first two. To be consistent, the random numbers associated with the first two modes are the same. Keeping ten modes instead of two means that the total variance explained rises from $\sim 92.73 \%$ to $\sim 98.97 \%$. The result is that, while the ensemble spread is enlarged in places, notably for the SSH in deep water in January, most of the time it is about the same as when only two modes are kept, and quite often it decreases. It seems that by adding the extra complexity to the wind perturbations we are cancelling out some of the variations we might otherwise produce. The higher order modes might just be adding statistical noise which doesn't represent the spatial pattern of the wind uncertainties, eventually leading to a decrease in spread.

With the ensemble EOF\|OBC we tested the second question by using the same wind perturbations that Vervatis et al. (2016) applied to generate the parent ensemble. We ensured for each member in the coastal ensemble that we matched its wind perturbations to the corresponding parent member which provided its open boundary conditions. Though the difference is small, the spread for this configuration is actually lower than that produced by our newly generated EOFs in the WIND $\times$ OBC ensemble. We generated another ensemble, EOFBISC, to measure the strength of these perturbations. This ensemble uses the same wind perturbations as EOF\|OBC but like the WIND ensemble keeps the unperturbed open boundaries for all members. The spread in SST is also almost always less than for the WIND ensemble, but 
the spread in SSH increases from the end of the ensemble spin-up period. This somewhat contradictory result demonstrates the difficulty in drawing general conclusions from these experiments - clearly a different set of EOFs will lead to a different set of wind perturbations which may be more or less successful at perturbing the ocean circulation model at different times.

As a final test, we configured yet another ensemble using the wind perturbations from the parent ensemble. The EOF $\perp$ OBC ensemble was configured the same as EOF $\|$ OBC except that the wind perturbations are no longer matched with the open boundary conditions from the equivalent member from the parent model. Instead, we forced each member's wind perturbation with the open boundary conditions from the member number two beneath it, effectively randomising them. The spread is higher than that of EOF $\|$ OBC for the whole period simulated, though still lower than that of the WIND $\times$ OBC ensemble. If anything, we must conclude that any resonance effect, should it exist, must be small, and that further perturbations (in this case generated by simply dissociating the perturbations from the open boundary conditions) can be expected to generate a greater spread rather than a lower one.

There are an infinite number of ways of generating perturbations, so we needed to make some choices. We made them based on the simple physical and intuitive assumption of generating wind uncertainties with a variability comparable to that of the wind itself. Despite other interesting questions, perhaps the most significant result for us from this series of experiments is that small variations to this method do not significantly change the pattern of model sensitivity, and hence do not change our conclusions.

\subsubsection{Experimenting with the nudging layer width}

A wider nudging layer ought to increase the influence of the parent model on the child model, preserving more of its behaviour in the downscaled system. But for an ensemble we expect the dynamics to be less impacted by the local perturbations (the wind, here). To learn something about the way the nudging layer width affects the ensemble spread we prepared three ensembles with a 10-point nudging layer instead of 30; these are WIND-NL10, OBC-NL10 and WIND $\times$ OBC-NL10. The narrower nudging layer was imposed after the ensemble spinup period, so the ensemble statistics are directly comparable with the other experiments. The time series of the ensemble spreads are shown in figures 6 and 7. Changing the nudging layer width has several repercussions, so we shall take the variables one at a time, starting with the SSH ensemble spreads. Overall, except for the first few days over the shelf and slope 
where the SSH is controlled mainly by non-local effects (the domain-wide, synchronous oscillations previously mentioned), the narrower nudging layer leads to greater spread for all three coastal ensembles. This is true even for the OBC run, despite there being no wind perturbations, doubtless a result of the greater freedom for the eddy dynamics. The WIND ensemble's spread sees the greatest relative increase from this modification, especially over the shelf and slope, where it even exceeds all the other ensembles for a few days in mid-March. The OBC and WIND $\times$ OBC ensembles react in a more complicated way, with the former's ensemble spread exceeding the latter's in deep water for most of the 3-month period. The reason for this isn't clear, but one possibility might be that the 5-day interval between random numbers (which sets up the time correlation of the wind perturbations) is insufficient, leading the wind perturbations to erode rather than strengthen the eddies. Over the slope and shelf, where local effects are more important, the WIND $\times$ OBC ensemble retains its supremacy.

For the SST things are again quite different. The WIND ensemble's spread is almost unchanged over the shelf, sometimes slightly lower, but in deep water it is higher everywhere with the narrower nudging layer. Here too, the reduced influence of the parent model allows the wind perturbations freer reign to spread the ensemble. But the OBC and WIND $\times$ OBC ensemble spreads are often weaker with the narrower nudging layer. Again, why this should be the case is not entirely clear, but one possibility is that while the WIND ensemble is less constrained by the parent model's unperturbed run, the other ensembles rely on the ensemble of open boundary conditions to generate their ensemble spread. Why this should have the opposite effect for SSH and SST may be explained by higher scalar diffusion in the coastal model as discussed in sections 5.3.1 and 5.3.2. With already smaller spread in SST because of the narrower nudging layer, the homogenisation of the ensemble's SST fields by the eddies might be more readily achieved.

These nudging layer experiments reinforce our observations from sections 5.3.1 and 5.3.2, that increasing SSH spread often comes at the cost of reducing the spread in SST. This is a trade-off which will have consequences for data assimilation schemes.

\subsubsection{The spatial structure of the ensemble spread of surface variables}

The plots in figures 6 and 7 give us useful mean quantities but to really understand how the perturbations affect the circulation it helps to look at some surface maps. Shown in figure 9 are, for the four principal ensembles, 
the time means of SSH ensemble spread for the three months following the ensemble spin-up phase. This compacted view of the ensembles over the period summarises their behaviour and major differences:

1. In all the ensembles the greatest contributor to the spread in SSH is from the mesoscale eddies. This follows from observing that the region where SSH ensemble spread is concentrated is also where the eddies are present.

2. These eddies are concentrated in deep water, with the continental slope (represented by the $200 \mathrm{~m}$ isobath) neatly circumscribing their range.

3. The WIND ensemble has the smallest spread, though in a concentrated area in the middle of the domain its ensemble spread is greater than that of the parent ensemble.

4. Over the shelf and at the domain's edge the WIND ensemble has the lowest spread. For the other ensembles, non-local processes contribute to the spread in these areas while the WIND ensemble is limited by only having one set of open boundary conditions.

5. The $\mathrm{OBC}$ and WIND $\times$ OBC ensembles are very similar-non-local errors dominate.

6. The ensemble spread of SSH is concentrated in the centre of the domain of the child ensembles, a feature not shared by the parent ensemble. One reason for this is the higher spatial coherence of the mesoscale eddies than in the parent model. Another possibility is that the eddies might be trapped in the coastal model's domain.

What this view doesn't show is the pronounced ensemble spread, seen as peaks in the time series in figure 6 , which results from the domain-wide pulsations generated in the parent model. It also doesn't show the comparable spread of the WIND ensemble to the others early in the period, before it falls off from mid-January. This is clearly visible in the downward trend of the WIND ensemble's deep-water SSH spread (the top-right graph in figure 6). It's an almost suspiciously smooth signal, but it seems to be a product of a particularity of the unperturbed run of the parent model (recall that all members of the WIND ensemble are forced at the open boundaries by the parent's unperturbed run). The large mesoscale eddy in that simulation shifts towards the northern and western boundaries of the BOBLAND domain during the simulation period. Being closer to the boundary means that it is more likely to be controlled by the prescribed open boundary conditions and is more resilient to being displaced by the wind. 
This behaviour is echoed in the ensemble means and spreads of the surface velocity fields in figures 10 and 11, respectively for the 20th of January and the 19th of February (representing the beginning and end of the downward trend in SSH spread). Comparing the two we see that the WIND ensemble's spread on the 20th of January is greater than a month later, and this reduction in ensemble spread manifests as a more spatially coherent eddy signal in the ensemble mean for the WIND ensemble in the February plot.

To lend support to this hypothesis we can compare with runs where we reduced the nudging layer from 30 points to 10 points (figure 6 , black and green dashed curves respectively). Sure enough, the effect of the wind perturbations on the SSH in this ensemble is less constrained by the open boundary conditions and the overall ensemble spread is higher. It still falls in February, but by proportionally less, and the recovery in March is stronger. Figure 12 shows surface maps for individual ensemble members for the 9th of March for the WIND and WIND-NL10 ensembles, where the latter is identical to the former except with the narrower 10-point nudging layer. By gathering all the member plots into one figure we are able to rapidly make a qualitative comparison (ignoring fine details) of the two ensembles. With the 30-point nudging layer, the large eddy barely changes position from member to member, whereas with a 10-point nudging layer the members are more distinct, with the eddy's position and strength varying much more. The 10-point nudging layer also allows the eddy's centre to get closer to the edge of the domain.

Let us return briefly to the velocity ensemble means and spreads in figures 10 and 11. Conspicuously absent from the spreads for the 20th of January (figure 10) is a signal corresponding to the slope current clearly visible in the zonal means. As this is true for the parent ensemble as well as for the coastal ensembles, this is an aspect of the parent model's dynamics that the coastal ensembles faithfully reproduce. Although we were able to find a weak spread at certain times, most of the time the slope current-when present - remained unmoved by the perturbations.

As with the SSH, we also produced plots for the time mean of surface temperature spread; these are shown in figure 13. The combination of different perturbation sources affects the SST's ensemble spread in similar ways to the SSH, though with some particularities. The salient points are:

1. Again the domain is divided into two zones by the continental slope, but here the ensemble spread is greatest over the shelf, with the spread 
produced by the river plumes dominating the signal.

2. In deep water, the WIND ensemble again has the lowest spread, and at the edges, where the spread is controlled by the open boundary conditions, the spread drops off; the parent ensemble has the highest spread.

3. Over the shelf, however, the WIND ensemble's spread is comparable to the other ensembles, with the river plume being the dominant source of error there.

4. As a consequence of this, the WIND $\times$ OBC ensemble's spread over the shelf is visibly greater than the OBC ensemble's, a finding reflected in the time series of spatial means of ensemble spread in figure 7 .

5. Meanwhile, it is the parent which has the largest spread over the shelf as it does overall, though with a different pattern which extends further south and west from the Adour's mouth and is weaker further north along the shelf.

6. The ensemble spread in the parent has localised peaks in contrast to the smoother pattern in the child model's ensembles.

\subsection{Beneath the surface}

In the preceding sections we've concentrated on the ensemble spread of surface variables, a natural place to look considering that the wind perturbations affect the surface directly and the ocean interior only indirectly. But there is a significant response to these perturbations deep beneath the surface too, in some places much stronger. Attributing an error response of the oceanic interior to surface forcing only is interesting enough from the standpoint of model and dynamic variability, but for data assimilation systems it suggests the possibility of correcting the model well away from the surface when surface data is assimilated. So with that in mind, in this section we explore the ensemble response in the coastal ocean interior.

\subsubsection{A general view of the ensembles beneath the surface}

We begin, once again, by concentrating on the four principal ensembles: WIND, OBC, WIND $\times$ OBC, and the parent ensemble. In all the ensembles the maxima of ensemble spread for salinity and temperature are either below the surface or over the continental shelf, under the influence of the river plume's wandering tendrils of cool, fresh water. Looking at time series of mean temperature spread near the base of the mixed layer (taken to be roughly 
$185 \mathrm{~m}$ below the surface), shown in figure 14, we note immediately that it is much greater than at the surface. We see that the spread of the parent is sometimes slightly higher than the other ensembles, but often considerably lower than all but the WIND ensemble. The slightly greater spread of parent relative to the coastal ensembles in the latter half of the period is mainly due to the deepening of the mixed layer, which puts the highly variable base beyond the depth at which we measured the statistics. A look at the vertical cross-sections in figure 15 shows a very high spread at the bottom of the mixed layer, which comes about as the depth of the thermocline changes in response to changes in mixing depth induced by the modified wind stress. This effect was also noted by Andreu-Burillo et al. (2002) following their sensitivity experiments. There are a couple of other conclusions we can draw immediately from figure 15: one is that from the surface to near the bottom of the mixed layer, but before reaching the thermocline, the parent ensemble's spread reduces more than the coastal ensembles', which remain fairly stable. This is consistent with our earlier conclusion of higher diffusion in the coastal model, but now it is manifested in the vertical direction. The second is that at the thermocline the spread of the coastal ensembles is greater than the parent's. Recalling that the coastal ensembles' higher spreads in SSH are reflected in their more coherent and stronger eddies, these would be expected to erode the surface stratification and deepen the mixed layer rather more than in the parent. This explains at least part of both observations, though it must be said that differences in the way that vertical mixing is parameterised would also be pertinent this close to the surface.

Once again, the equivalent plots for salinity do not add enough new information to warrant their inclusion here. Suffice it to say that deep below the surface the effect of the river plumes is very small.

At the increased depths of $500 \mathrm{~m}$ and $1000 \mathrm{~m}$, shown in figure 16, the parent ensemble spread is still lower again than the coastal ensembles, though it still overtakes the WIND ensemble later in the time series. This behaviour too is visible in figure 15. Below the surface mixed layer, the coastal ensembles feature a strong spread which coincides with a second, deeper thermocline. At the same depth the parent ensemble's spread almost disappears. The vertical gradients of temperature and salinity are very similar in both parent and coastal models, so we may once more attribute this difference to the more detailed small-scale dynamics of the coastal model.

Arguably it is here, below the mixed layer, that the coastal model distinguishes itself the most from the regional parent model. The enhanced hori- 
zontal resolution allows it to resolve small-scale eddies (as small as $\sim 10 \mathrm{~km}$ in diameter), and the sigma-level vertical discretisation allows for a more detailed description of the circulation's interaction with bottom relief. As a result, not only are the eddies stronger and penetrate deeper, but from about a thousand metres below the surface the coastal model's horizontal velocity fields become wonderfully complex, almost belying any connection to the parent model's dynamics. Figure 17 illustrates this for the meridional velocity component for unperturbed runs of the parent and coastal models at the surface, and three horizontal sections at different depths. The velocity fields are stronger in the coastal model at all depths, but that's not sufficient to explain the fall at depth in the parent's ensemble spread relative to the coastal model. In deep water, the surface velocity fields are dominated by the large, anticyclonic mesoscale eddy, with a smaller cyclonic one adjoining it on its south-eastern edge (clear in the coastal model, and hinted at in the parent model). At $500 \mathrm{~m}$ these eddies still dominate the velocity field, but at $1000 \mathrm{~m}$ they fracture into smaller, less coherent structures. This change in regime isn't unique to the coastal model, but what differentiates it from the parent model is the high density of variation. While the large structures dominate at the surface for both models, in deeper water it is the smaller structures, which only the coastal model resolves, which dominate. Some of this complexity arises from small eddies generated by the flow around the underwater gorges on the continental slopes, which shows the importance not only of the model's higher resolution but of the bathymetry's too, as well as the terrain-following vertical sigma coordinates.

\subsubsection{The reduced nudging layer's effects at depth}

As we did for section 5.4.1, we plotted curves for the coastal ensembles with a reduced nudging layer at depth (figures 14 and 16 green curves). The results below the surface are less ambiguous than for the surface, if one remembers to take into account that in the latter part of the period the mixed layer deepens, and the ensemble statistics start to resemble the surface more than the thermocline. Apart from this, the reduced nudging layer leads to a greater ensemble spread, consistent with the coastal model's stronger response to perturbations at depth. Freed somewhat from the constraints of the parent model, it develops even more differences between members as we descend the water column. 


\subsubsection{Interaction of eddies with bottom near a strong gradient}

We noticed a particularly strong spread at a specific time and place which we thought worthy of closer examination. In figure 18 we show cross-sections of the WIND ensemble centred on an eddy. The unperturbed run's SSH is depicted in figure 18a, in which an eddy dipole can be made out.

Figure 18b shows a cross-section of ensemble spread of temperature, and several features stand out. Firstly, there is a large spread at the base of the surface mixed layer, as discussed in section 5.4.1. There is also a small region of larger spread at the coast, a result of the wind pushing the cool, fresh water plume of the Adour in different directions, and it being mixed through the water column. But most interestingly, there is a region between 1000 and $1500 \mathrm{~m}$ depth and -3 and $-2.5^{\circ}$ longitude with high ensemble spread. It is particularly strong along the gently sloping Landes Plateau. This feature is all the more remarkable because of the region of reduced spread between it and the surface mixed layer, and at these depths the vertical mixing produced by surface stresses cannot have much influence - neither, in fact, can the open boundary conditions, since only local wind perturbations have been applied. The unperturbed temperature profile in figure 18c shows the second, deep thermocline mentioned in section 5.4.1. It lies beneath a region of much gentler temperature gradient which extends from the near-surface thermocline to a depth of around $1200 \mathrm{~m}$. The zonal velocity field at this depth, plotted in figure 18e, shows that the eddy visible in the SSH signal in figure 18a extends down to just about reach the slope.

The final plot in the figure, 18f, shows the spread of the zonal velocity. While it decreases with depth, it is still significant near the second thermocline. This and the anomalously high values of vertical velocity spread shown in figure 18d suggest that interactions between the bottom and the eddy are responsible for the enhanced spread in temperature. The vertical velocity spread is especially high at the bottom where it coincides with the high spread in temperature. There is meanwhile a very small ensemble spread in vertical velocity coinciding with the near-surface thermocline. This is telling: the thermocline is a region of strong temperature gradient, and therefore high stability, where we would expect vertical movement to be suppressed. We can hence differentiate the mechanism which displaces the near-surface thermocline from that which produces the strong temperature spread near the bottom, where strong vertical movements are present within the stable layer. Oey and Zhang (2004) showed that warm eddies interacting 
with a slope could lead to big modifications to the deep circulation, including cyclones and jets. While in this particular case we do not see substantial alterations to the horizontal velocities, the vertical velocities do appear to be affected by such an interaction.

At other times not shown, vertical motion (and consequently its spread) is suppressed in the deep thermocline as well even when there is a large spread of temperature. And there are doubtless other complex processes leading to enhanced spread that a more exhaustive study of the ensembles could identify. We can say with certainty that the presence of the eddy-slope interaction is not necessary to generate a large spread deep beneath the surface. Over the deep abyssal plain of the Bay of Biscay, Vervatis et al. (2016) demonstrated this by assimilating SSH data, which were able correct the strong errors between 1000 and $1500 \mathrm{~m}$. Ayoub et al. (in prep.) also observed a high spread at these depths far from the slope. But the eddy-slope interaction provides another mechanism by which model errors can be generated at depth and, hence, by which the model can be corrected at depth by data assimilation at the surface. The use of the coastal model is decisive here, because although we could not verify whether or not the vertical velocities were affected in the parent model, there is no such corresponding enhanced spread in temperature. It is possible that the eddy-slope interaction is present, but the result is barely, if at all, discernible. Interestingly, it is the WIND ensemble which shows the strongest spread here, and by some way. Even the WIND $\times$ OBC ensemble doesn't come close, resembling more closely the OBC ensemble. This isn't then a question of pure strength of perturbation, but of a confluence of factors: the right perturbation leading to the right deviations of the eddy with the right temperature gradient in the right place so that the conditions are favourable for the eddy-slope interaction to generate the spread in temperature.

\subsection{Extent and diffusion of river plumes}

Only the Adour river runoff is explicitly modelled in the coastal model, but the Gironde's runoff is also modelled in the parent model. Its plume enters the coastal model from the northern boundary, forming as it does, with the Adour's plume, a significant body of cool, less saline water. As we noted in section 5.3.5, the behaviour of the Adour plume is quite different in the two models, leading to different ensemble statistics.

Direct comparison between the models is complicated, because even though the prescribed outflow volumes of the Adour are the same for most of 
the time period in the BISCAY36 and BOBLAND configurations, the way that river water is injected into the Bay of Biscay is different. Both use a single grid point as the source but this grid point is much smaller in BOBLAND than in BISCAY36. The depth at the river mouth is also different, with BISCAY36 drawing its depth from its coarse bathymetry, about $10.5 \mathrm{~m}$, and BOBLAND using a prescribed depth of $5 \mathrm{~m}$. The areas of the outward faces of the grid cells are then about $22950 \mathrm{~m}^{2}$ and $2500 \mathrm{~m}^{2}$ respectively, so the discharge velocity is more than 9 times greater in BOBLAND than in BISCAY36. A higher velocity could produce a longer plume, but could also generate more local turbulence and generate more mixing, dissipating the plume earlier. The salinity is treated in a similar way in the two river models: it is prescribed and insignificant compared to the salinity levels in the open ocean (0 PSU in BOBLAND and 0.1 PSU in BISCAY36). Temperature, on the other hand, is handled differently, with only minimum and maximum values prescribed in BOBLAND $\left(10^{\circ}\right.$ and $\left.22^{\circ}\right)$ with the seasonal temperature cycle set with a sinusoid. In BISCAY36 the temperature is determined from a Neumann boundary condition, with the river discharge grid point treated as an open boundary. With these differences, only a gross evaluation of the river plume behaviour between the two configurations would be appropriate, but between the three different BOBLAND ensembles we can be more concrete.

Our analysis is based on using the surface salinity as a proxy for the river plume. For each member of an ensemble, we calculated the area of each plume, whose extents were defined by one of three thresholds. Taking the ensemble mean, we may determine a typical plume area for each model. These areas are shown plotted over time in figure 19.

The parent model clearly has the most expansive plume by all three of the threshold definitions, with only occasional exceptions, mainly at the beginning of the time series. Interestingly, the plumes from the three coastal ensembles are very similar; to the extent that they do differ from one another, they do not follow the pattern of ensemble spreads of surface temperature and salinity. As described in section 5.3 and figure 7 (recall that the surface salinity and temperature behave almost identically), these spreads are by far the greatest in the parent ensemble, followed by the WIND $\times$ OBC and OBC ensembles (these two are very close) and then the WIND ensemble, with by far the least spread. However although the parent ensemble's plume area is on average greater, the next greatest is, more often than not, the OBC ensemble, with the WIND $\times$ OBC ensemble usually found between it and the WIND ensemble. The greater spread of the parent ensemble seems to reflect 
its broader average plume, but between the coastal ensembles things are a bit subtler. A greater spread in SST or SSS in one coastal ensemble than in another could mean that since the plume is displaced further, it is also diffused more effectively. This threshold value is hence attained closer to the source. This is just one factor in a complicated process.

To shed more light on the plume's behaviour, we examine the salinity and its spatial gradients for the parent and child models, shown in figure 20 . The example shown is for the first perturbed members of the WIND $\times$ OBC and parent ensembles. It appears as though we can distinguish three salinity regimes in both models: (1) the inner, fresh zone near the river mouth, with a strong gradient as the fresh water mixes quickly with the surrounding saline water; (2) an intermediate zone of mixed water distinct from the main, outer circulation; and (3) the saline, outer circulation. The inner zone of the coastal model has a very strong gradient at the river mouth, representing a rapid mixing of the plume here, while the parent's mixing is slower. By the time we get to the edge of this zone, however, the parent model's plume stabilises and remains coherent for longer, and the coastal model's intermediate zone is patchy and diffuse. Even though a direct comparison is impossible, the ocean colour observations of Petus et al. (2014) do favour the coastal model's representation. The very sharp boundary in the observations between the river plume and the surrounding water is situated very close to the river mouth, closer in character to the more localised and more quickly diffused plume of the coastal model.

\section{Discussion and conclusions}

We sought to downscale a region model ensemble by forcing a coastal model ensemble by an ensemble of open boundary conditions, as well as by local wind perturbations. We found that, overall, the ensemble of open boundary conditions coupled with local wind perturbations generated the greatest spread in the coastal model, but that the contribution from the open boundary ensemble was greater than the local wind perturbations, and the combination of the two led to only a slightly greater spread. It seems as though the non-local errors advected into the model were more important in generating local errors than the local wind perturbations. It was only during the early stages of the ensemble spinup, before the non-local perturbations had time to penetrate into the domain, did we see the local wind perturbations generate a greater spread over the shelf. Another contributing factor 
could be that although the OBC ensemble was generated without local wind perturbations, the perturbed surface characteristics resulting from the nonlocally produced errors creates a difference in each member's wind stress. This might partly explain why an additional wind perturbation doesn't add too much to the coastal ensemble's spread.

There was a distinct difference between the signals over the shelf and over deep water for all the ensembles. The SSH spread was usually greater over deep water, where the forcing perturbations displaced the large mesoscale eddy; the SST spread was greater over the shelf, where the river plume dominated the signature. Conversely, the SST spread was lower over deep water and the SSH spread was for most of the period lower over the shelf, the exception being the large, non-locally produced synchronous events which had an outsized effect over shallow waters. The behaviour is different too, with the spread of both variables over the shelf varying spatially and temporally more so than over deep water.

We experimented with different methods of generating the wind perturbations and combining them with the ensemble of open boundary conditions. Interestingly, the exact form of the wind perturbations did not have a huge impact on the shape of the time series - the magnitude changed significantly, but periods of large spread and low spread were mostly conserved. This suggests that the intrinsic variability of the model dominates over any specific perturbation scheme. This may also explain the saturation in spread that appeared to occur near the end of the ensemble spinup period with the different wind perturbations.

Of course this is not quite as true when we switch from an ensemble with only local wind perturbations to one forced by the parent ensemble at the open boundaries. In this case, the non-local errors can be related to much larger scale processes that the coastal model alone cannot represent, whatever the local perturbation scheme. The dominance of intrinsic variability and the insensitivity to the precise form of errors in boundary and initial conditions has been noted in geophysical modelling before, for example by Giorgi and Bi (2000) and Rinke et al. (2004). We must be careful not to draw too many similarities with our own experiments - for one thing, not all of their conclusions (which include insensitivity to perturbation magnitude) apply to our ensembles. But it seems safe to conclude that the finer details in a wind perturbation scheme are of relative unimportance.

The choice of nudging layer had consequences for the ensemble spread, critically so for the WIND ensemble. With a broad nudging layer, the large 
mesoscale eddy was present in the same area near the edge of the domain for all members of the WIND ensemble. This lead to a sustained drop in ensemble spread, an effect which disappeared almost entirely with application of the narrower nudging layer. The narrower nudging layer also led to a bigger spread in SSH for the other coastal ensembles, even though their spreads in SST saw only modest and inconsistent increases.

One of the more intriguing results in the present work is how deep subsurface errors can be generated by surface perturbations. A large spread at the base of the seasonal thermocline was anticipated, having been previously described (Andreu-Burillo et al., 2002). But for there to be such a strong signal at much greater depths, beyond $1000 \mathrm{~m}$, was less expected. To be sure, the parent model had a measurable spread there too, but while the signals at the near-surface thermocline were similar for the parent and coastal ensembles, the deep water spread was in places at least twice as strong in the coastal ensembles than in the parent ensemble. This suggests a valuable contribution that the downscaling paradigm can make to the study of model errors. It is especially appealing from a data assimilation perspective, for if we can generate deep, subsurface errors then we might hope to be able to correct these regions with only surface data. The vertical temperature gradients for the two models' unperturbed runs, we noted, were almost the same, but the model and bathymetry resolutions were much higher in the coastal model. A major consequence of this is the more detailed dynamics, of course, but more specifically it is the ability of the coastal model to resolve more coherent mesoscale eddies with stronger currents. These can reach deeper in the coastal model than in the parent model, whose less coherent eddy has a much weaker effect at these depths. We also saw that eddy-slope interactions could be an additional mechanism for ensemble divergence, something we did not see in the parent ensemble. The use of sigma layer depth coordinates in the coastal model may have contributed to this behaviour.

The river plume's behaviour was very different between the parent and child models, and this contributed to the parent model's mostly higher SST spread. Among the coastal ensembles the spread was usually greatest for the OBC ensemble, though the difference was small. According to our threshold criterion, the mean plume area does not appear to be directly correlated with the ensemble spread. We did find that the plume's behaviour supported our hypothesis that the parent model diffuses scalars less than the higher resolution coastal model, which accounted for some of the differences in spread between the parent ensemble and the child ensembles. 
A recurring feature of the ensembles is that the WIND $\times$ OBC ensemble did not always have a greater spread than the OBC ensemble. Logically, more sources of perturbation should lead to more spread, as we can explore more regions of the error space. But with a limited number of ensemble members we cannot always guarantee an increase in spread as we pile on perturbations. This limitation could probably be somewhat alleviated by increasing the magnitude of the perturbations, but our aim is to not simply increase the spread at any cost - we are interested in the model's response to forcing errors, and we would prefer the error space to be densely populated by our ensemble. Despite the sometimes lower spread, the benefit of adding an extra forcing perturbation is that it opens the error spaces which may be left untouched, or weakly affected, by any one forcing perturbation. We can see this in our ensembles. Figure 13 shows almost no spread at the edge of the continental shelf for the coastal ensembles, but the spread, while still weak, is slightly enhanced when wind perturbations are added.

The next phase of this work will be to use what we have learned in a data assimilation context, in which we interpret the ensemble spread as the model's uncertainty. Of course a successful data assimilation scheme requires that the ensemble's spread span the data-Vervatis et al. (in review, 2019) propose empirical techniques to determine this. We hope to present these results in a future paper.

\section{Acknowledgements}

We would like to thank Cyril Nguyen and Thomas Duhaut of the Laboratoire d'Aérologie in Toulouse for their help with running and adapting the Symphonie model, and Florent Lyard of LEGOS for helping us with the tidal analysis and for supplying the high-resolution bathymetry. We would also like to thank Patrick Marchesiello for useful discussions on model dissipation. This project was granted access to the HPC resources of the CALMIP supercomputing centre through grants P1119 and P1325 for the 2016-2017 allocations, and thanks go to Emmanuel Courcel, Nicolas Renon and Pierrette Barbaresco for their support. Support from Didier Gazen and Laurent Cabanas of the Laboratoire d'Aérologie, Toulouse, for the use of the Nuwa cluster is also gratefully acknowledged. This research was undertaken as part the AMICO project and was supported by the GMES-MDD joint program of the French Ministère de la Transition écologique et solidaire and CNRS/INSU. 


\section{References}

van Aken, H.M., 2000. The hydrography of the mid-latitude Northeast Atlantic Ocean: II: The intermediate water masses. Deep Sea Research Part I: Oceanographic Research Papers 47, 789-824. doi:10.1016/S09670637(99)00112-0.

Andreu-Burillo, I., Caniaux, G., Gavart, M., De Mey, P., Baraille, R., 2002. Assessing ocean-model sensitivity to wind forcing uncertainties. Geophysical Research Letters 29, 5.1-5.4. doi:10.1029/2001GL014473.

Auclair, F., Marsaleix, P., De Mey, P., 2003. Space-time structure and dynamics of the forecast error in a coastal circulation model of the Gulf of Lions. Dynamics of Atmospheres and Oceans 36, 309-346. doi:doi:10.1016/S0377-0265(02)00068-4.

Ayoub, N., De Mey, P., Marsaleix, P., in prep. Constraining a coastal ocean model by SST and SSH observations using an Ensemble Kalman Filter in the Bay of Biscay (North-East Atlantic) .

Echevin, V., De Mey, P., Evensen, G., 2000. Horizontal and vertical structure of the representer functions for sea surface measurements in a coastal circulation model. Journal of Physical Oceanography 30, 2627-2635.

Estournel, C., Auclair, F., Lux, M., Nguyen, C., Marsaleix, P., 2009. "Scale oriented" embedded modeling of the North-Western Mediterranean in the frame of MFSTEP. Ocean Science , 73-90.

Estournel, C., Zervakis, V., Marsaleix, P., Papadopoulos, A., Auclair, F., Perivoliotis, L., Tragou, E., 2005. Dense water formation and cascading in the Gulf of Thermaikos (North Aegean) from observations and modelling. Continental Shelf Research , 2366-2386doi:doi:10.1016/j.csr.2005.08.014.

Giorgi, F., Bi, X., 2000. A study of internal variability of a regional climate model. Journal of Geophysical Research: Atmospheres 105, 29503-29521.

Griffies, S.M., Hallberg, R.W., 2000. Biharmonic friction with a Smagorinsky-like viscosity for use in large-scale eddy-permitting ocean models. Monthly Weather Review 128, 2935-2946. 
Herbert, G., 2012. Modélisation et observation de la dynamique haute fréquence de la circulation du Golfe de Gascogne. Ph.D. thesis. Université de Toulouse.

Herbert, G., Ayoub, N., Marsaleix, P., Lyard, F., 2011. Signature of the coastal circulation variability in altimetric data in the southern Bay of Biscay during winter and fall 2004. Journal of Marine Systems 88, 139158.

Jordà, G., De Mey, P., 2010. Characterization of error dynamics in a 3D coastal model of the Catalan sea using stochastic modelling 30, 419-441.

Katavouta, A., Thompson, K.R., 2016. Downscaling ocean conditions with application to the Gulf of Maine, Scotian Shelf and adjacent deep ocean. Ocean Modelling 104, 54-72. doi:10.1016/j.ocemod.2016.05.007.

Kersalé, M., Marié, L., Cann, B.L., Serpette, A., Lathuilière, C., Le Boyer, A., Rubio, A., Lazure, P., 2016. Poleward along-shore current pulses on the inner shelf of the Bay of Biscay. Estuarine, Coastal and Shelf Science 179, 155-171. doi:https://doi.org/10.1016/j.ecss.2015.11.018. special Issue: Functioning and dysfunctioning of Marine and Brackish Ecosystems.

Kim, S., Samelson, R.M., Snyder, C., 2011. Towards an uncertainty budget for a coastal ocean model. Monthly Weather Review 139, 866-884.

Kourafalou, V.H., De Mey, P., Staneva, J., Ayoub, N., Barth, A., Chao, Y., Cirano, M., Fiechter, J., Herzfeld, M., Kurapov, A., Moore, A.M., Oddo, P., Pullen, J., van der Westhuysen, A., Weisberg, R.H., 2015. Coastal ocean forecasting: science foundation and user benefits. Journal of Operational Oceanography 8, s147-s167. doi:10.1080/1755876X.2015.1022348, arXiv:https://doi.org/10.1080/1755876X.2015.1022348.

Large, W.G., Yeager, S.G., 2004. Diurnal to Decadal Global Forcing for Ocean and Sea-Ice Models: The Data Sets and Flux Climatologies. Technical Report. NCAR Technical Note NCAR/TN-460+STR.

Le Cann, B., 1990. Barotropic tidal dynamics of the Bay of Biscay shelf: observations, numerical modelling and physical interpretation. Continental Shelf Research 10, 723-758. 
Le Cann, B., Serpette, A., 2009. Intense warm and saline upper ocean inflow in the southern Bay of Biscay in autumn-winter 2006-2007. Continental Shelf Research 29, 1014-1025.

Le Hénaff, M., De Mey, P., Marsaleix, P., 2009. Assessment of observational networks with the Representer Matrix Spectra method-application to a 3D coastal model of the Bay of Biscay. Ocean Dynamics , 320doi:10.1007/s10236-008-0144-7.

Le Sommer, J., Penduff, T., Theetten, S., Madec, G., Barnier, B., 2009. How momentum advection schemes influence current-topography interactions at eddy permitting resolution. Ocean Modelling 29, 1-14.

Lucas, M.A., Ayoub, N., Barnier, B., Penduff, T., De Mey, P., 2008. Stochastic study of the temperature response of the upper ocean to uncertainties in the atmospheric forcing in an Atlantic OGCM. Ocean Modelling 20, 90-113. doi:10.1016/j.ocemod.2007.07.006.

Lyard, F., 2015. private communication.

Madec, G., the NEMO team, 2008. NEMO ocean engine. Note du Pôle de modélisation 27. Institut Pierre-Simon Laplace (IPSL), France. ISSN No 1288-1619.

Maraldi, C., Chanut, J., Levier, B., Ayoub, N., De Mey, P., Reffray, G., Lyard, F., Cailleau, S., Drevillon, M., Fanjul, E.A., Sotillo, M.G., Marsaleix, P., the Mercator Research, Team, D., 2013. NEMO on the shelf: assessment of the Iberia-Biscay-Ireland configuration. Ocean Science , 745-771doi:10.5194/os-9-745-2013.

Marchesiello, P., McWilliams, J.C., Shchepetkin, A., 2001. Open boundary conditions for long-term integration of regional oceanic models. Ocean Modelling 3, 1-20.

Marsaleix, P., Auclair, F., Estournel, C., 2006. Considerations on open boundary conditions for regional and coastal ocean models. Journal of Atmospheric and Oceanic Technology , 16041613doi:http://dx.doi.org/10.1175/JTECH1930.1. 
Marsaleix, P., Auclair, F., Estournel, C., 2009a. Low-order pressure gradient schemes in sigma coordinate models: The seamount test revisited. Ocean Modelling , 169-177doi:http://dx.doi.org/10.1016/j.ocemod.2009.06.011.

Marsaleix, P., Auclair, F., Estournel, C., Nguyen, C., Ulses, C., 2011. An accurate implementation of the compressibility terms in the equation of state in a low order pressure gradient scheme for sigma coordinate ocean models. Ocean Modelling , 113doi:http://dx.doi.org/10.1016/j.ocemod.2011.07.004.

Marsaleix, P., Auclair, F., Estournel, C., Nguyen, C., Ulses, C., 2012. Alternatives to the Robert-Asselin filter. Ocean Modelling , 5366doi:http://dx.doi.org/10.1016/j.ocemod.2011.11.002.

Marsaleix, P., Auclair, F., Floor, J.W., Herrmann, M.J., Estournel, C., Pairaud, I., Ulses, C., 2008. Energy conservation issues in sigma-coordinate free-surface ocean models. Ocean Modelling , 6189doi:http://dx.doi.org/10.1016/j.ocemod.2007.07.005.

Marsaleix, P., Ulses, C., Pairud, I., Herrmann, M.J., Floor, J.W., Estournel, C., F., A., 2009b. Open boundary conditions for internal gravity wave modelling using polarization relations. Ocean Modelling , 2742doi:http://dx.doi.org/10.1016/j.ocemod.2009.02.010.

Oey, L.Y., Zhang, H.C., 2004. The generation of subsurface cyclones and jets through eddy-slope interaction. Continental Shelf Research 24, 2109-2131. doi:10.1016/j.csr.2004.07.007. recent Developments in Physical Oceanographic Modelling: Part I.

Orain, F., 2016. Product user manual for Level 3 SST products over European Seas. Technical Report.

Pairaud, I.L., Auclair, F., Marsaleix, P., Lyard, F., Pichon, A., 2010. Dynamics of the semi-diurnal and quarter-diurnal internal tides in the Bay of Biscay. part 2: Baroclinic tides. Continental Shelf Research , 253269doi:10.1016/j.csr.2009.10.008.

Pairaud, I.L., Lyard, F., Auclair, F., Letellier, T., Marsaleix, P., 2008. Dynamics of the semi- diurnal and quarter-diurnal internal tides in the bay of biscay. part 1: Barotropic tides. Continental Shelf Research , 12941315doi:doi:10.1016/j.csr.2008.03.004. 
Petus, C., Marieu, V., Novoa, S., Chust, G., Bruneau, N., Froidefond, J.M., 2014. Monitoring spatio-temporal variability of the adour river turbid plume (bay of biscay, france) with modis 250-m imagery. Continental Shelf Research 74, 35-49.

Pingree, R.D., Le Cann, B., 1992. Three anticyclonic Slope Water Oceanic eDDIES (SWODDIES) in the Southern Bay of Biscay in 1990. Deep-Sea Research 39, 1147-1175.

Puillat, I., Lazure, P., Jégou, A.M., Lampert, L., Miller, P., 2006. Mesoscale hydrological variability induced by northwesterly wind on the French continental shelf of the Bay of Biscay. Scientia Marina , 15-26.

Rinke, A., Marbaix, P., Dethloff, K., 2004. Internal variability in Arctic regional climate simulations: case study for the SHEBA year. Climate research 27, 197-209.

Rubio, A., Fontán, A., Lazure, P., González, M., Valencia, V., Ferrer, L., Mader, J., C., H., 2013. Seasonal to tidal variability of currents and temperature in waters of the continental slope, southeastern Bay of Biscay. Journal of Marine Systems , S121-S133doi:10.1016/j.jmarsys.2012.01.004.

Sandery, P.A., Sakov, P., 2017. Ocean forecasting of mesoscale features can deteriorate by increasing model resolution towards the submesoscale. Nature Communications 8. doi:10.1038/s41467-017-01595-0.

Solabarrieta, L., Rubio, A., Castanedo, S., Medina, R., Charria, G., Hernández, C., 2014. Surface water circulation patterns in the southeastern Bay of Biscay: New evidences from HF radar data. Continental Shelf Research , 60-76.

Toublanc, F., Ayoub, N.K., Lyard, F., Marsaleix, P., Allain, D.J., 2018. Tidal downscaling from the open ocean to the coast: a new approach applied to the Bay of Biscay. Ocean Modelling 214, 16-32. doi:https://doi.org/10.1016/j.ocemod.2018.02.001.

Toumazou, V., Cretaux, J.F., 2001. Using a Lanczos eigensolver in the computation of empirical orthogonal functions. Monthly Weather Review 129, 1243-1250. doi:10.1175/1520-0493(2001)129¡1243:UALEIT¿2.0.CO;2. 
${ }_{1347}$ Vervatis, V., Testut, C.E., De Mey, P., Ayoub, N., Chanut, J., Quat${ }_{1348}$ trocchi, G., 2016. Data assimilative twin-experiment in a high1349 resolution Bay of Biscay configuration: 4DEnOI based on stochas1350 tic modeling of the wind forcing. Ocean Modelling 100, 1-19. 1351 doi:http://dx.doi.org/10.1016/j.ocemod.2016.01.003.

1352 Vervatis, V.D., De Mey-Frémaux, P., Ayoub, N., Sofianos, S., Testut, C.E., ${ }_{1353}$ Kailas, M., Karagiorgos, J., Ghantous, M., in review, 2019. Assessment ${ }_{1354}$ of a NEMO-based physical-biogeochemical stochastic model in a high1355 resolution Bay of Biscay configuration. Geoscientific Model Development 1356 


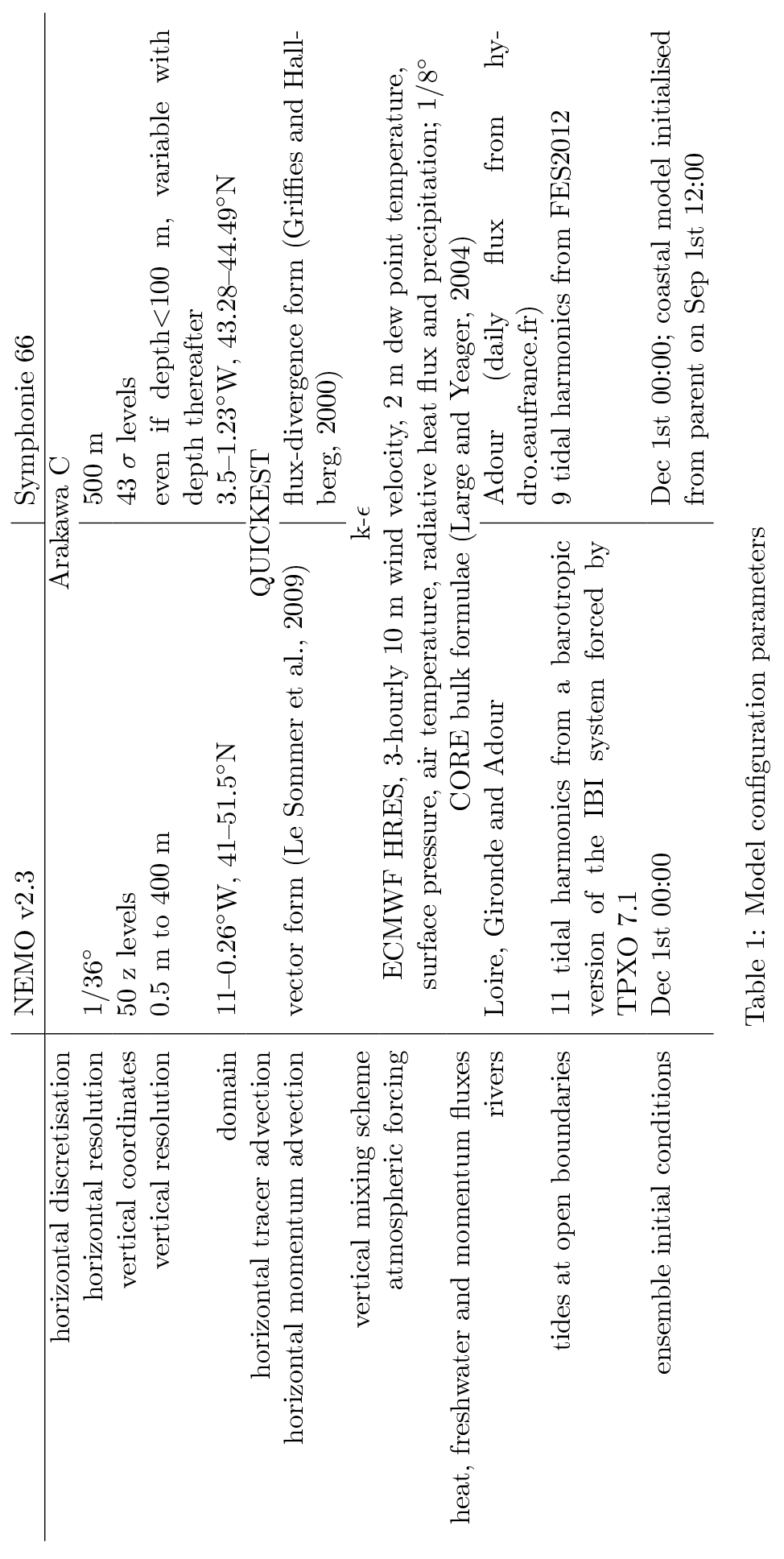




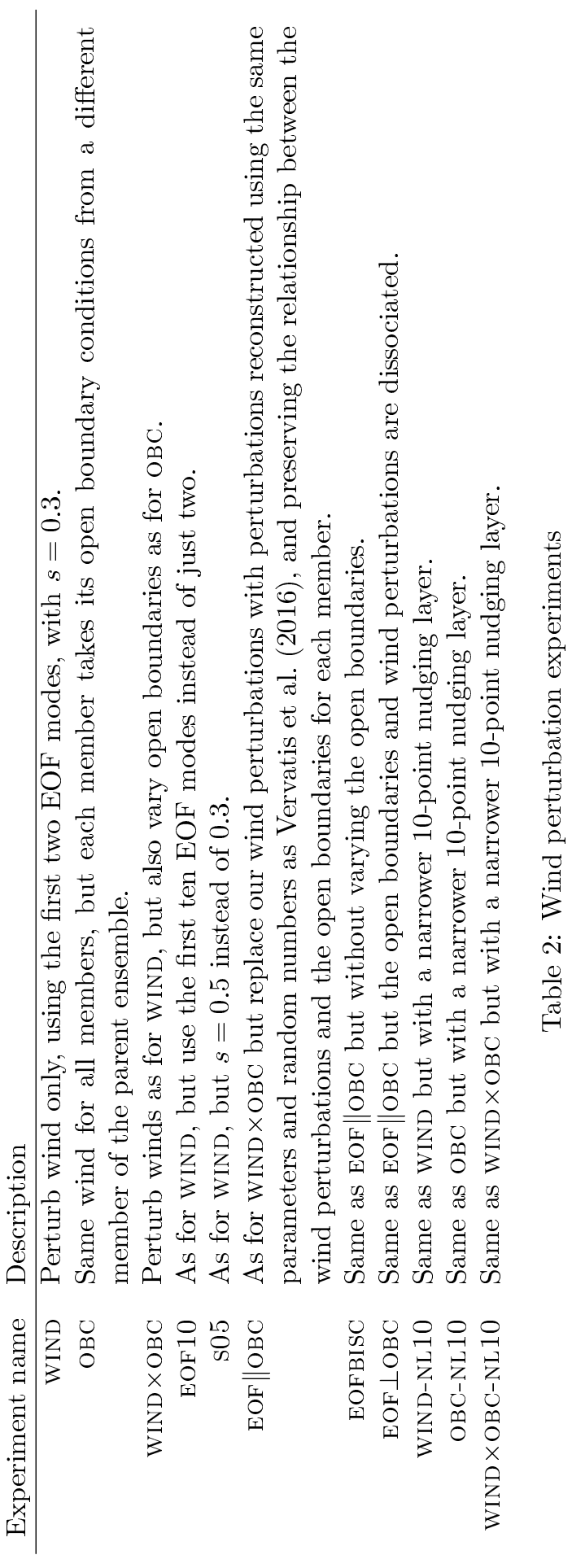




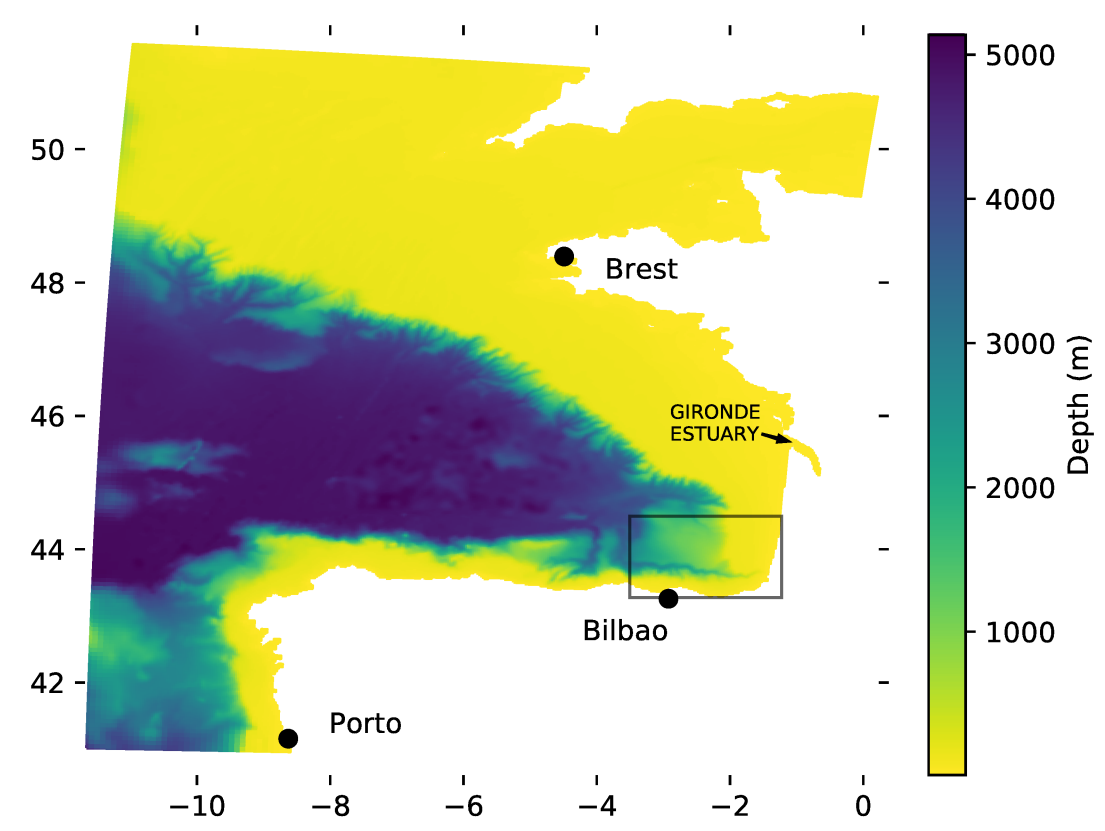

Figure 1: Bathymetry of the regional parent model. The black square shows the coastal domain. 


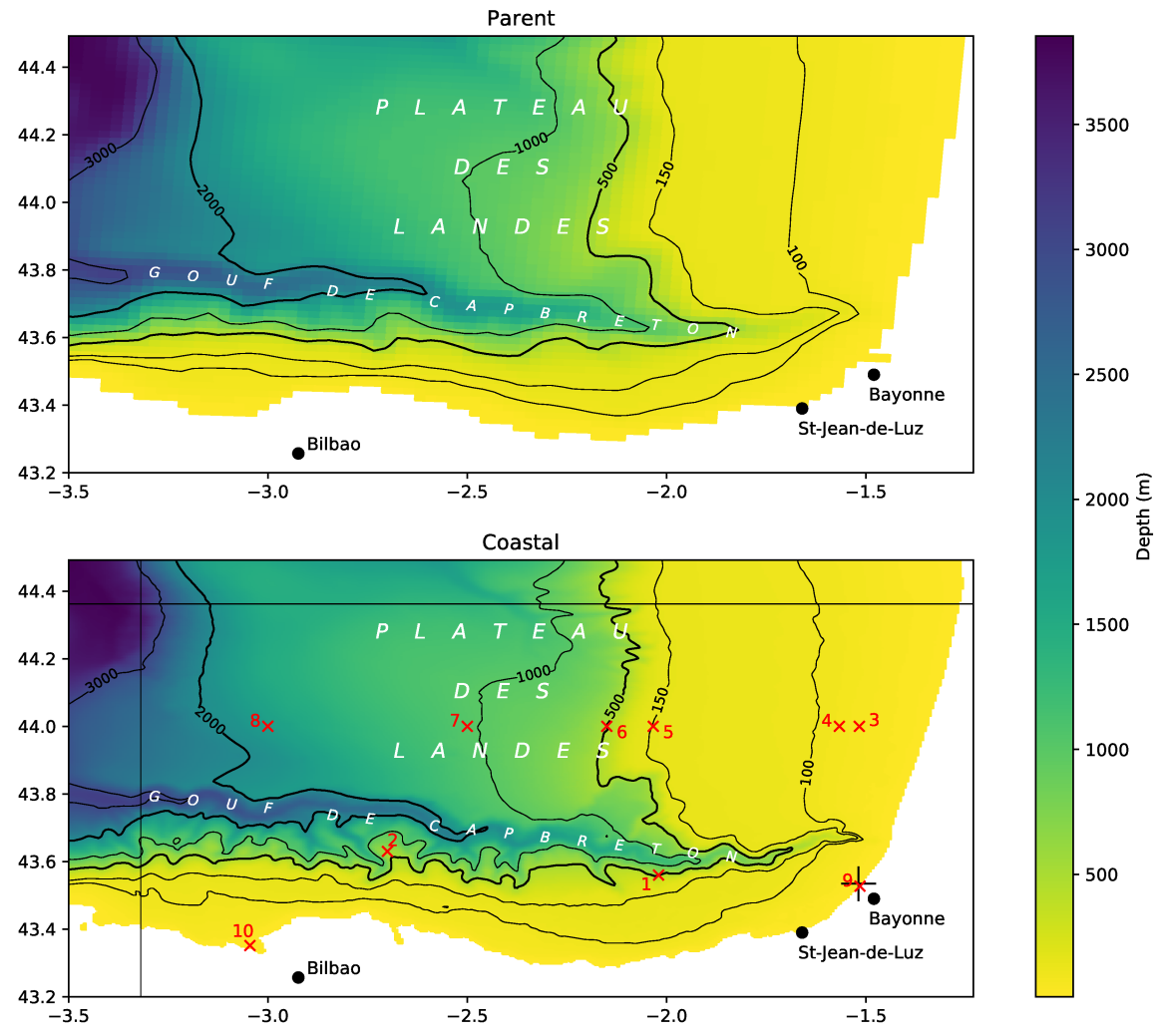

Figure 2: Bathymetries used in the regional parent model (top) and the coastal child model (bottom) shown as they are interpolated onto the model grids in the coastal domain. The numbered crosses show the locations of the high frequency time series used in section 5.2 and figure 5. The vertical and horizontal lines show the edge of the coastal model's 30point nudging layer. The large, black cross indicates the location of the Adour's discharge in the child model; the notch in the land mask above Bayonne in the parent locates the river discharge there. 
Wind temporal std dev.; EOFs calculated over coastal domain
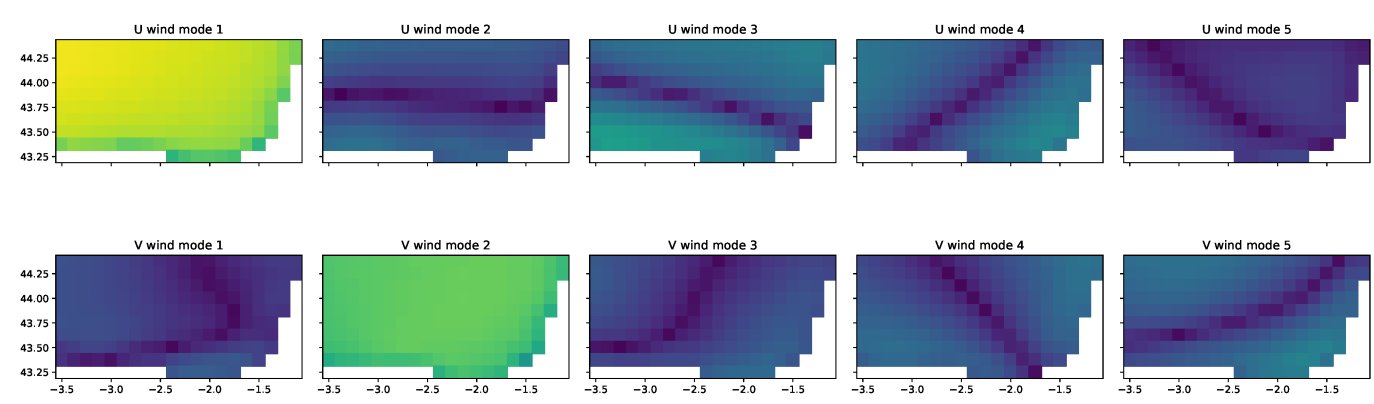

$\checkmark$ wind mode 2
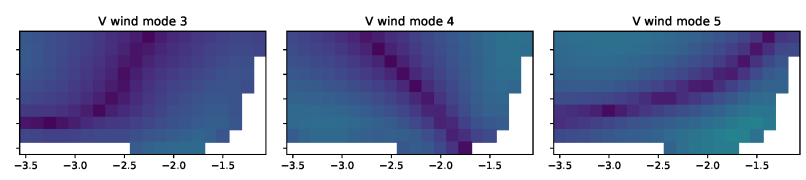

Figure 3: Temporal standard deviations of the first five modes of the wind EOFs for the BOBLAND configurations over the four month period of the ensemble runs. Except for the EOF10ensemble, only the first two modes were used to generate coastal ensembles.

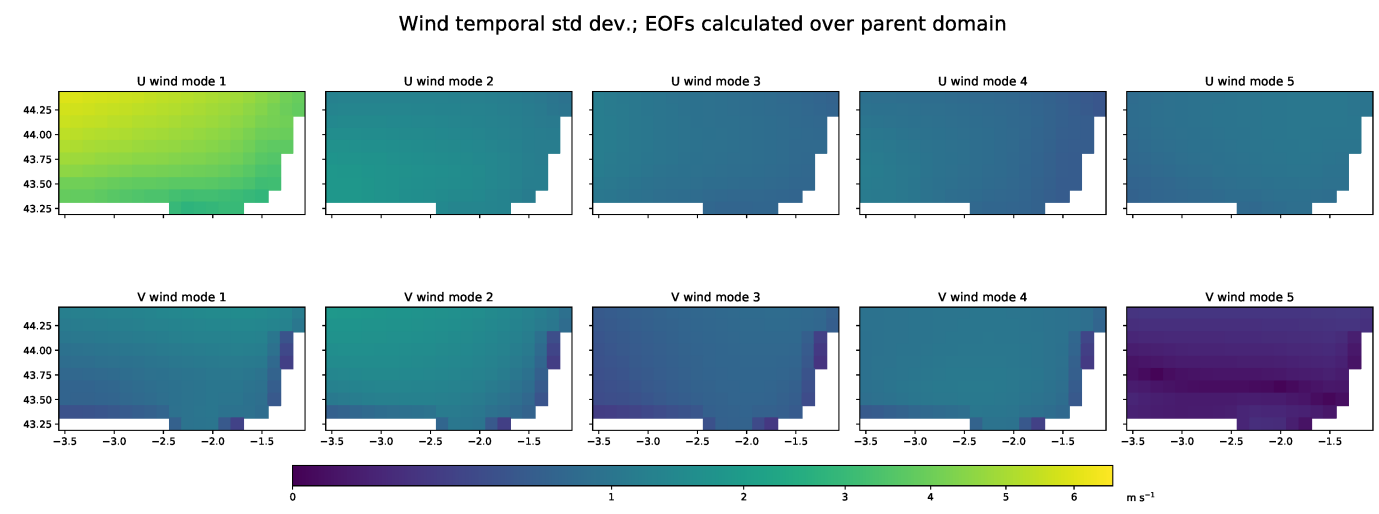

Figure 4: Temporal standard deviations of the first five modes of the reconstructed wind EOFs for the BISCAY36 model configuration, over the four month period of the ensemble runs. 


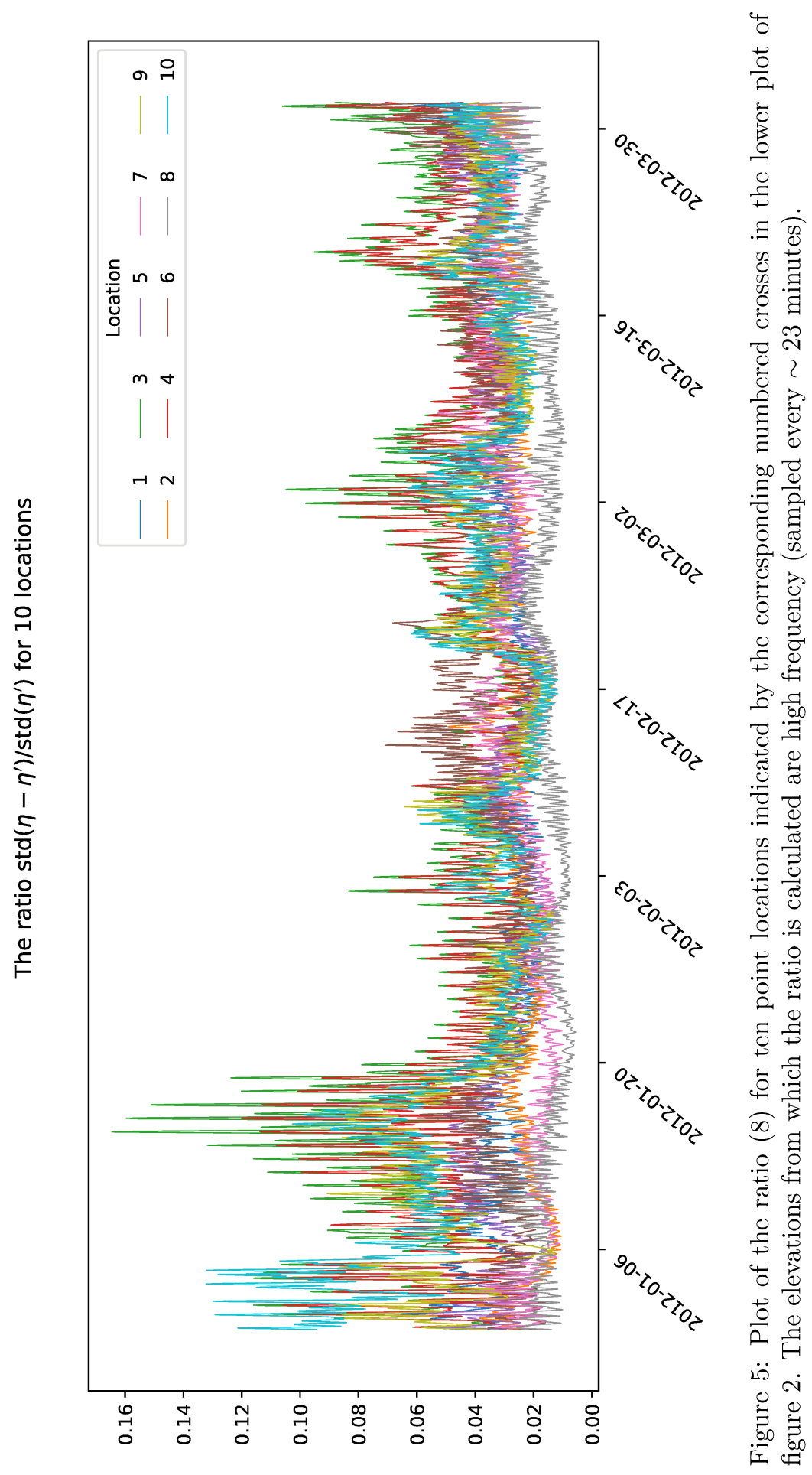



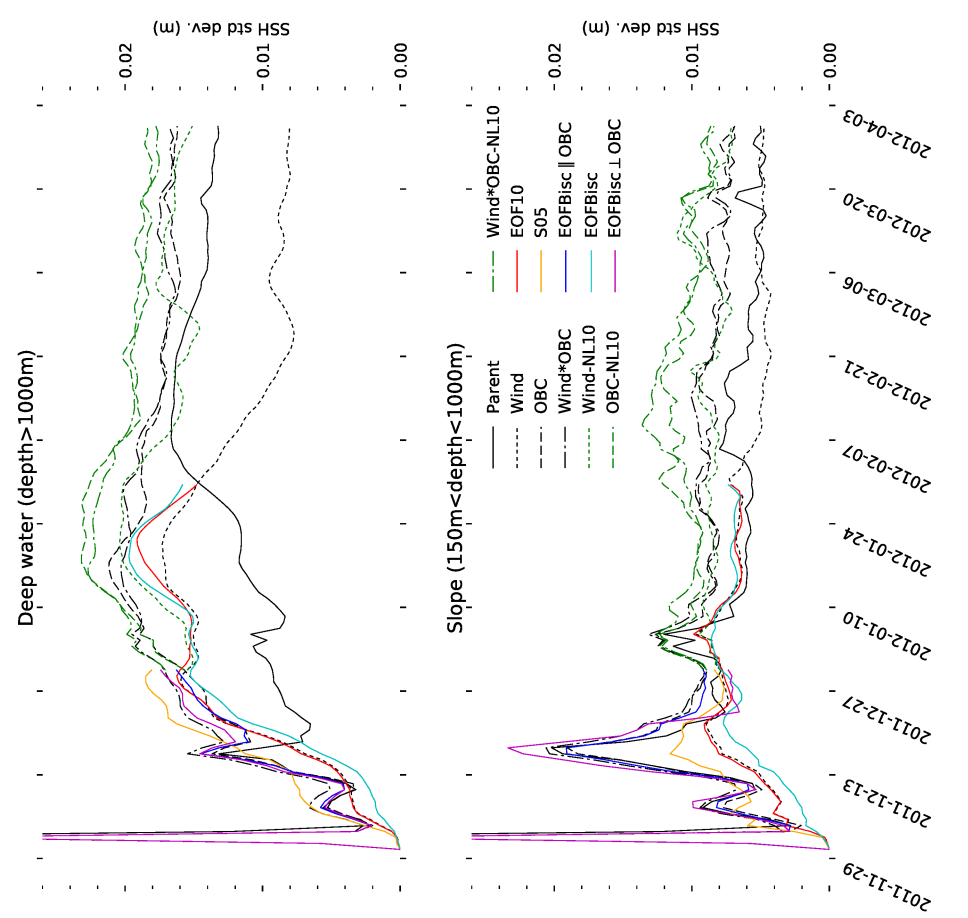

을 苦营

웅

额

韋宫苛

ปี

苛

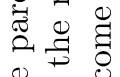

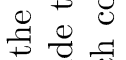

译:

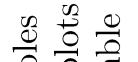

글

券券

하

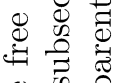

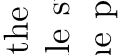

tᄒ

田

की
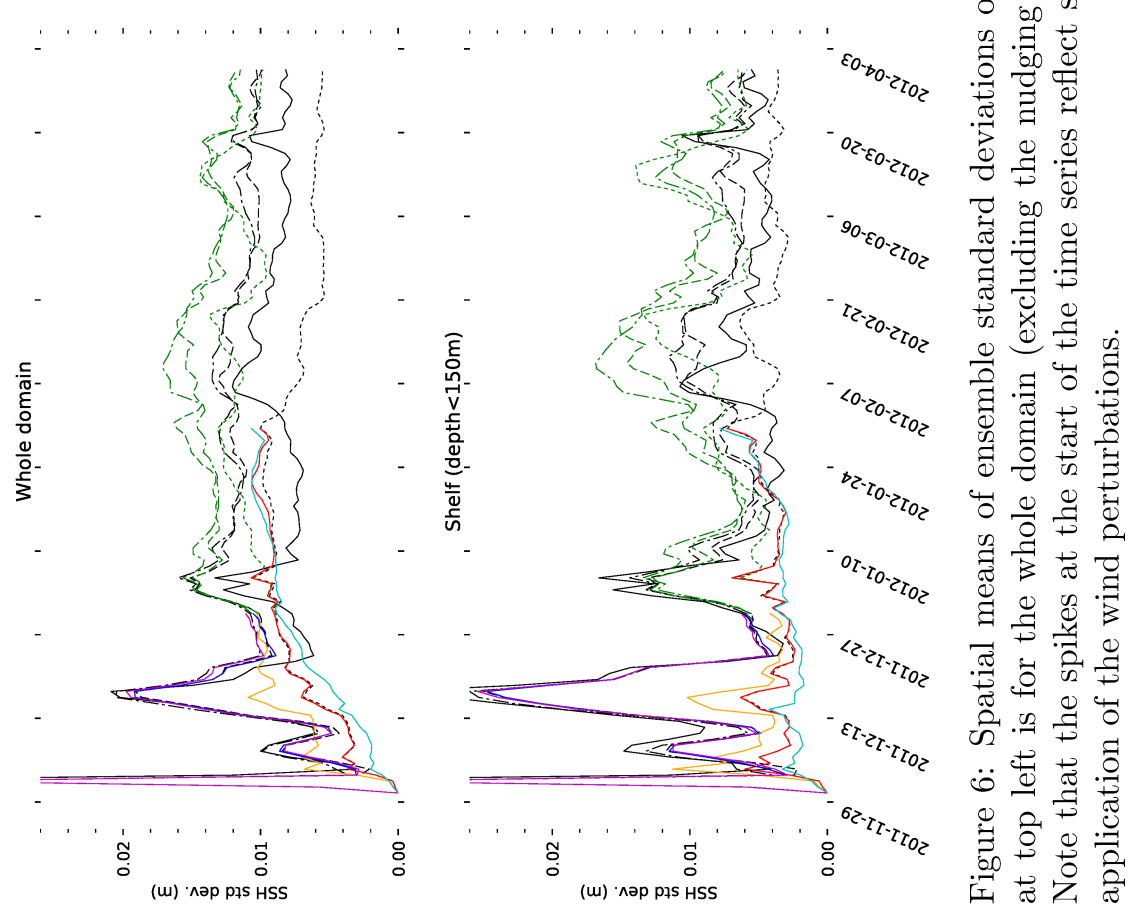


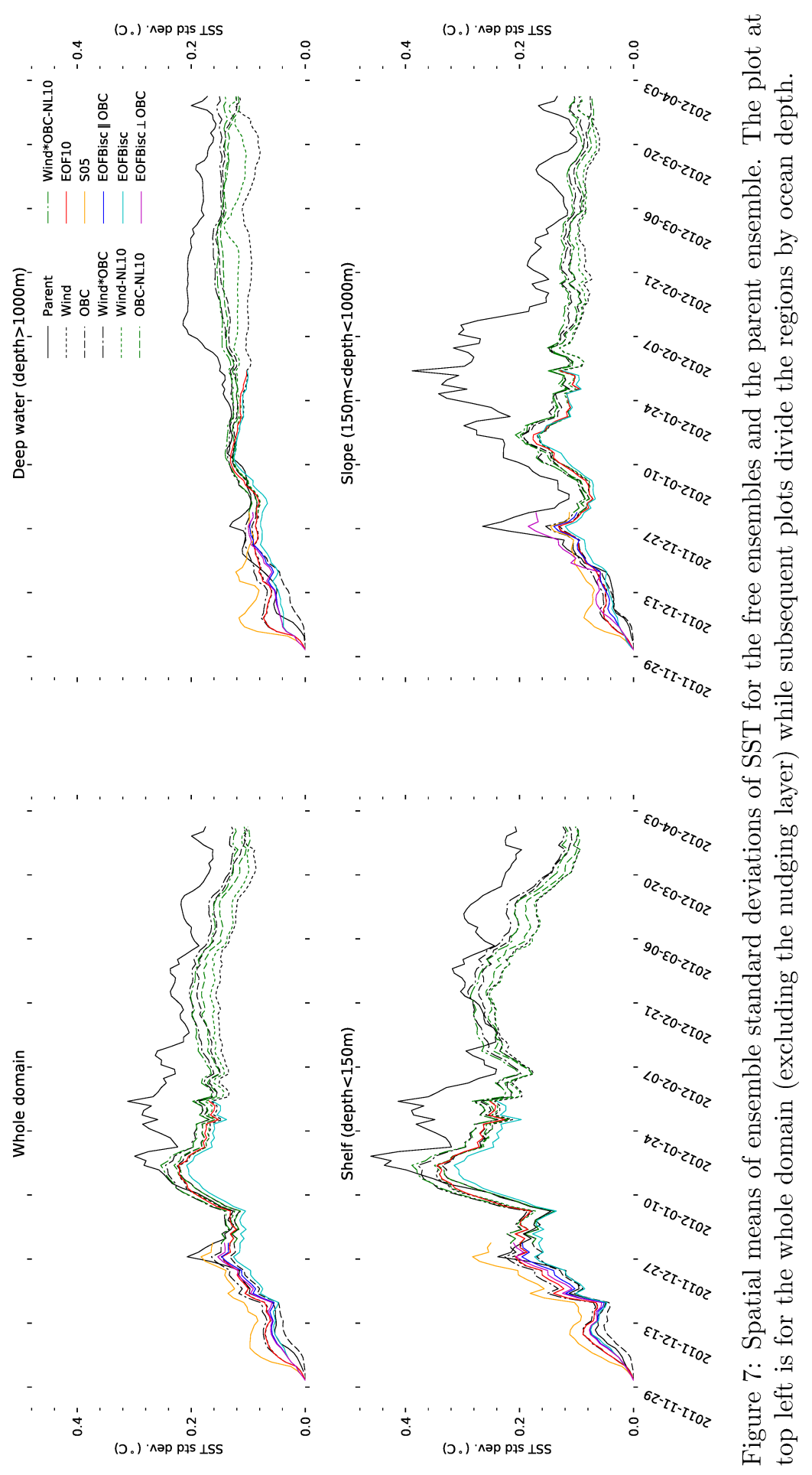




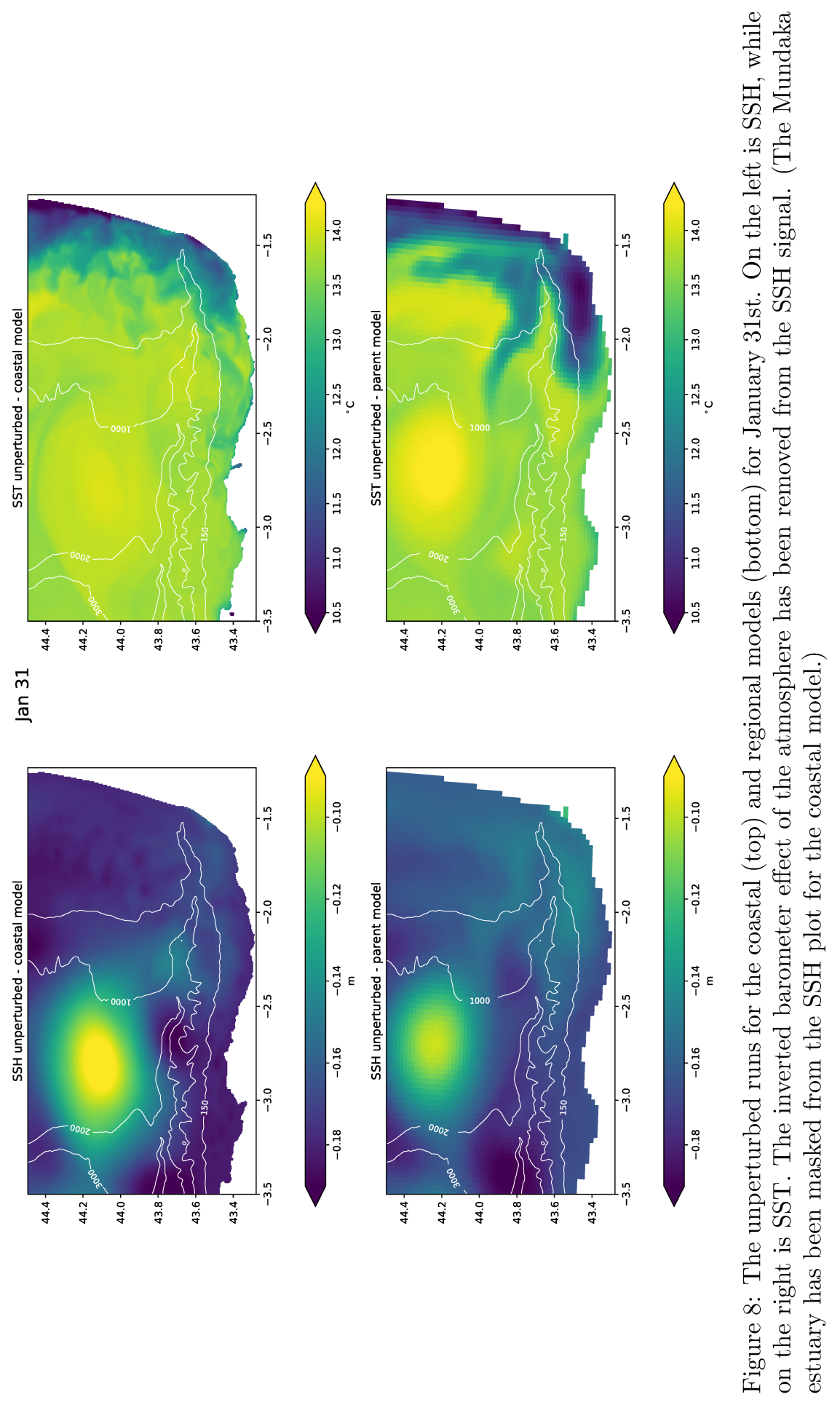




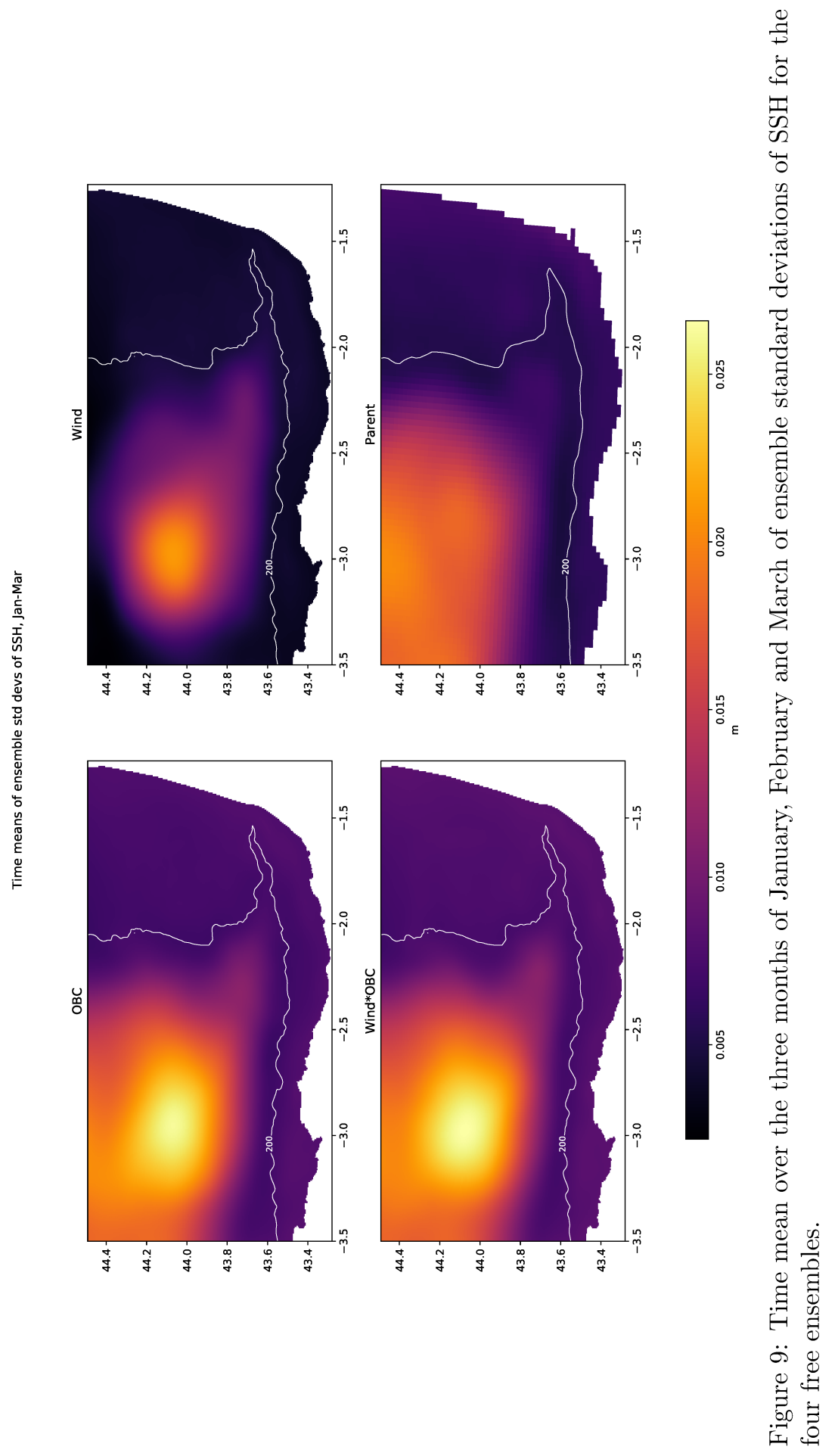




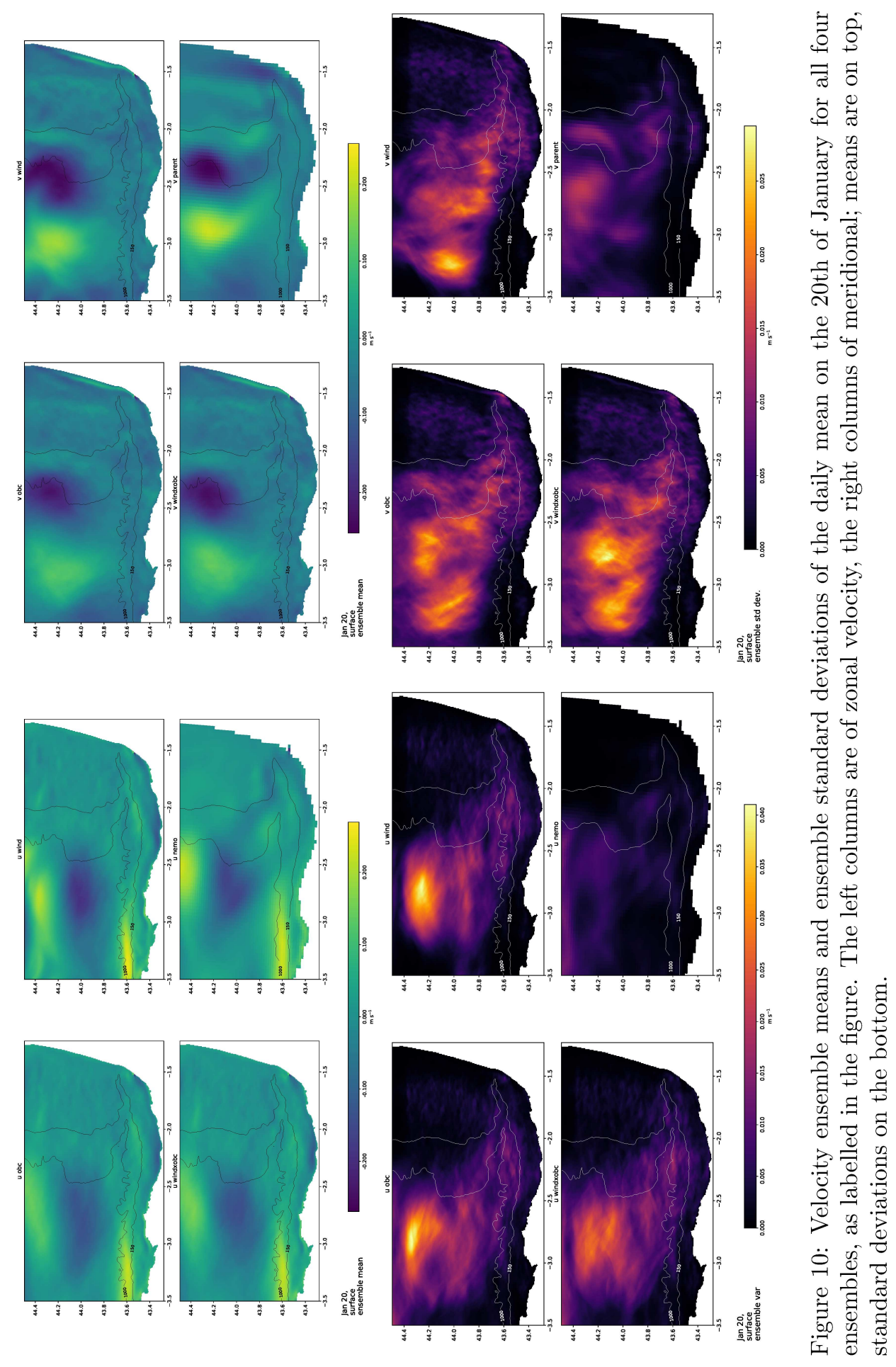




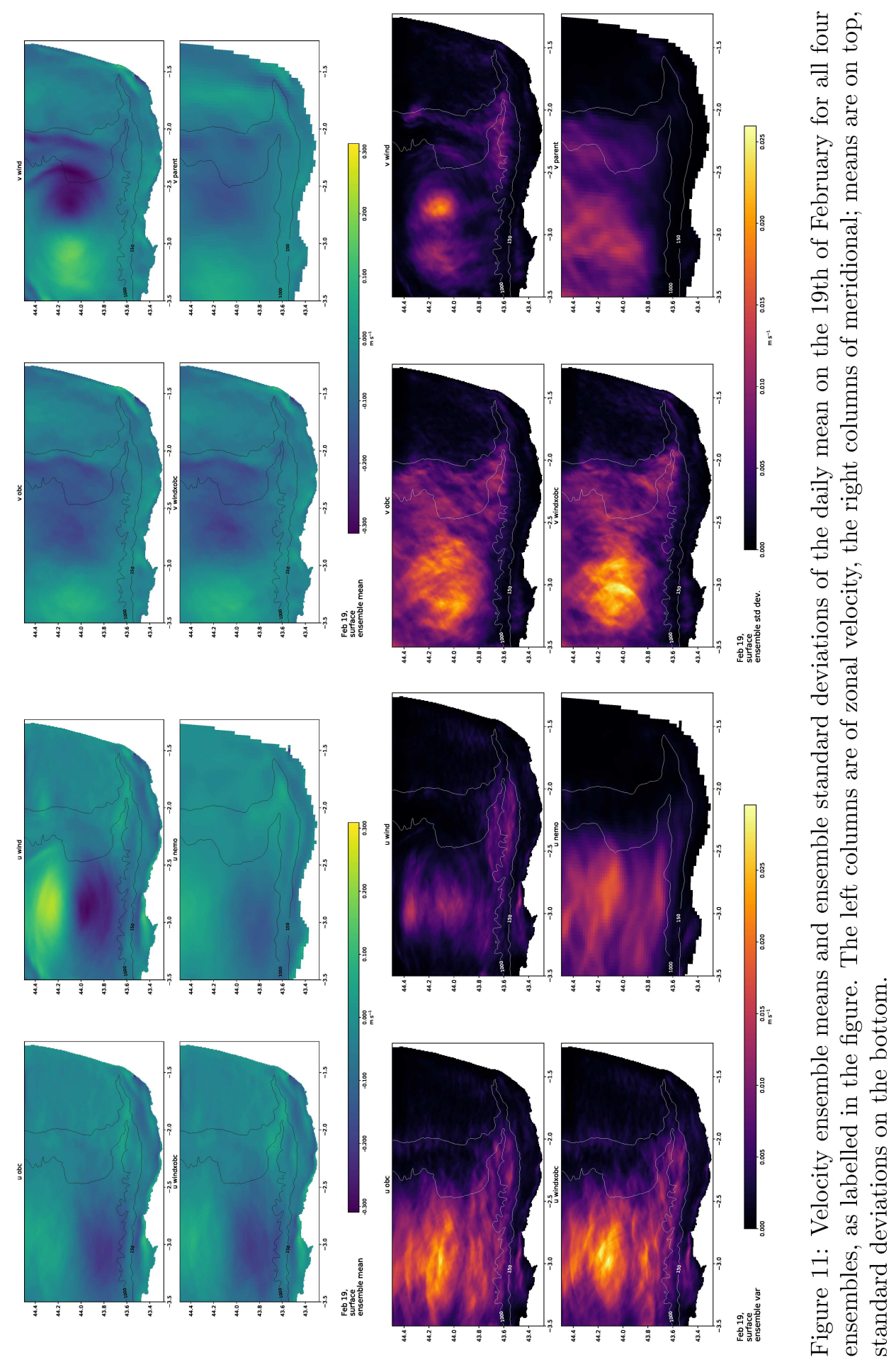




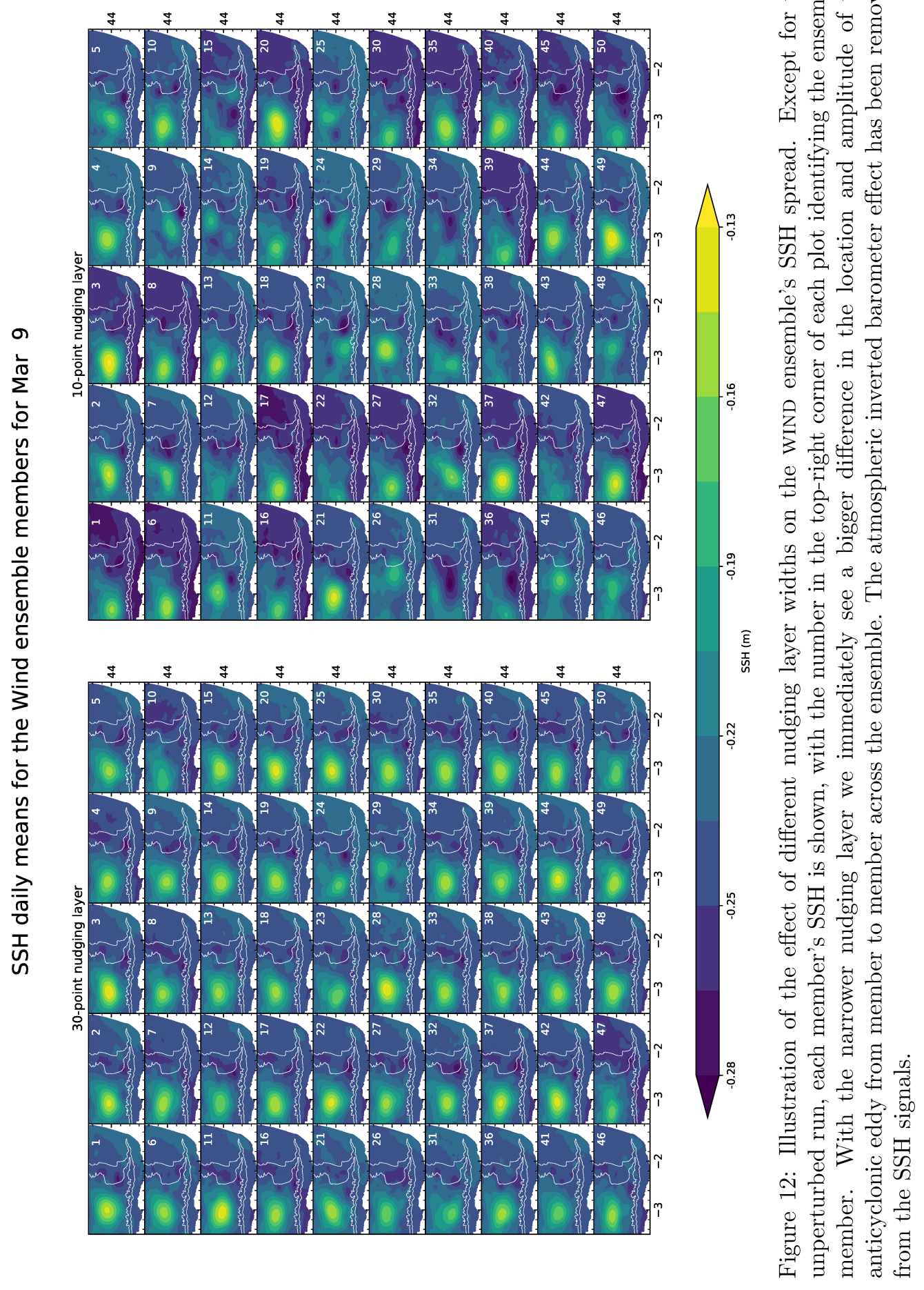




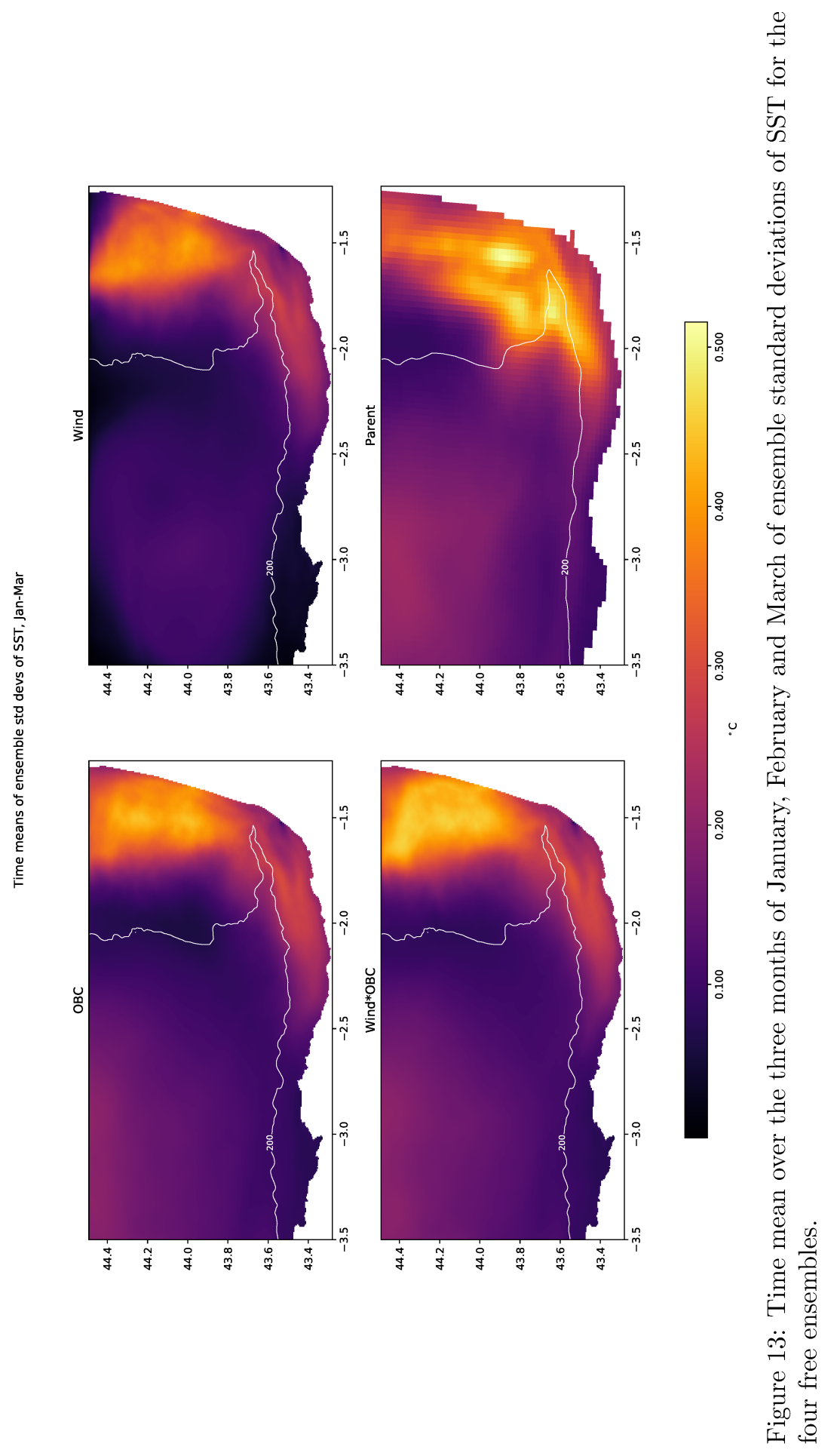




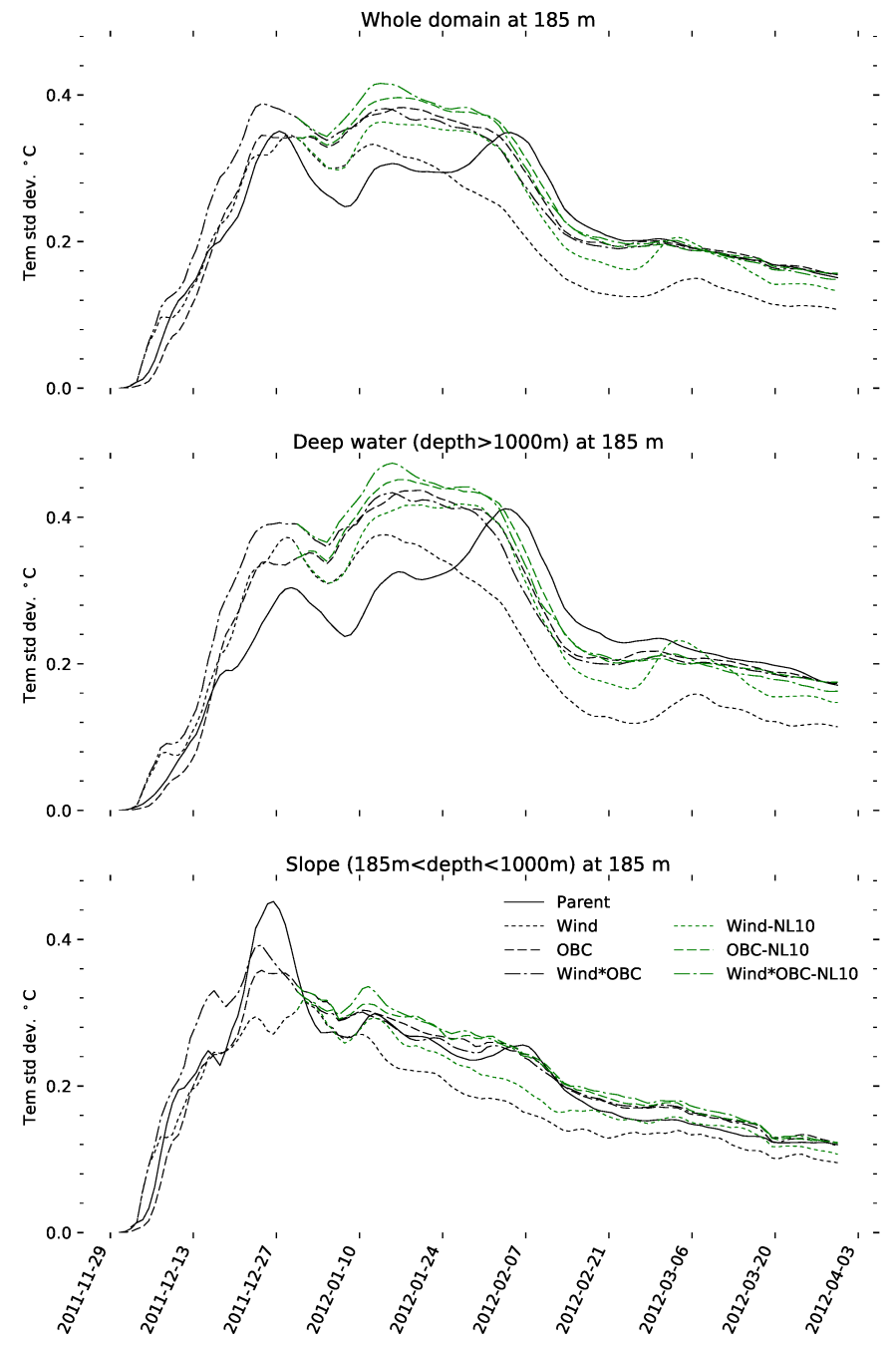

Figure 14: Spatial means of ensemble standard deviations of temperature on a horizontal slice at $185 \mathrm{~m}$ depth for the free ensembles and the parent ensemble. The plot at top left is for the whole domain (excluding the nudging layer) while subsequent plots divide the regions by ocean depth. 


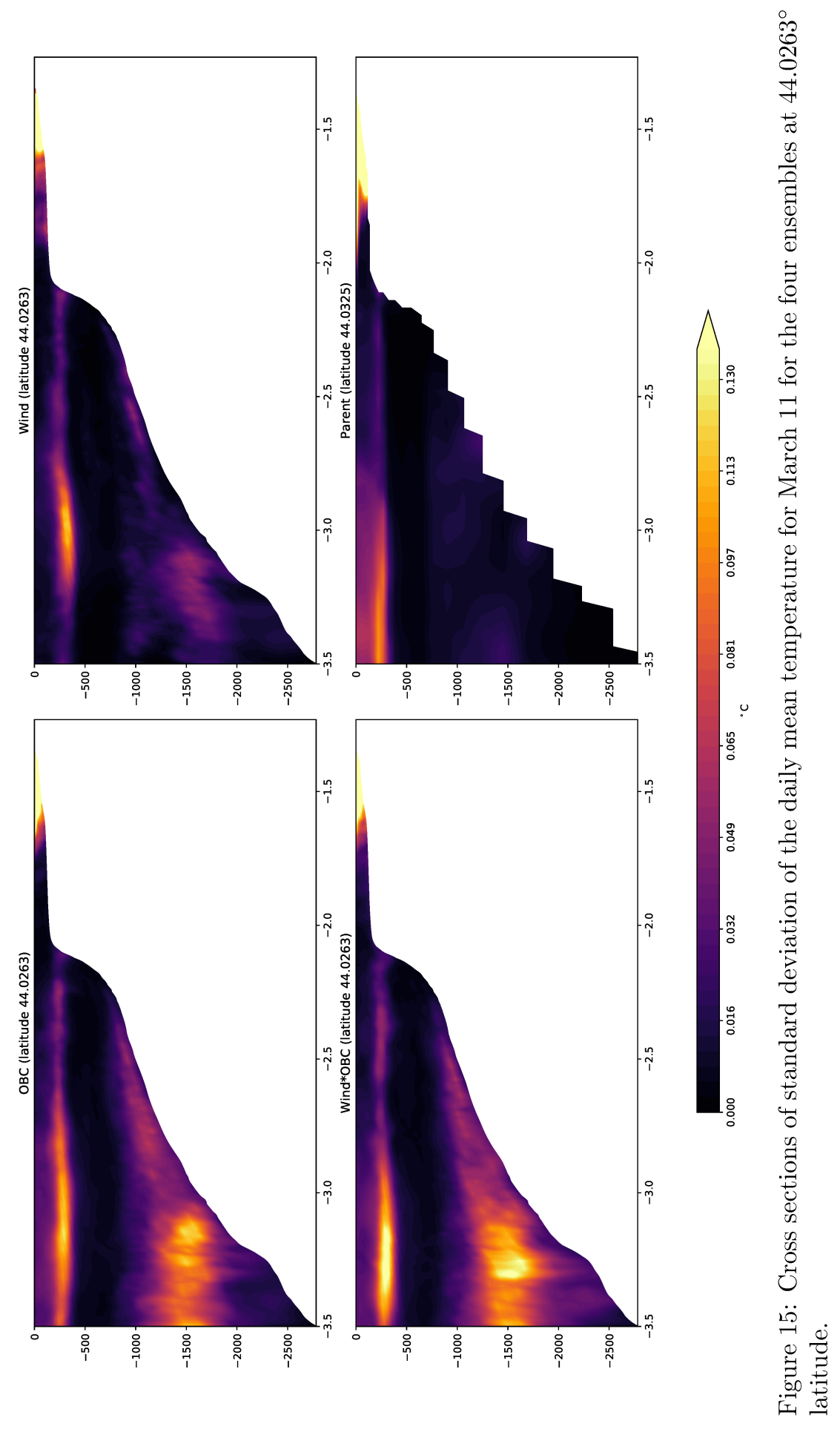




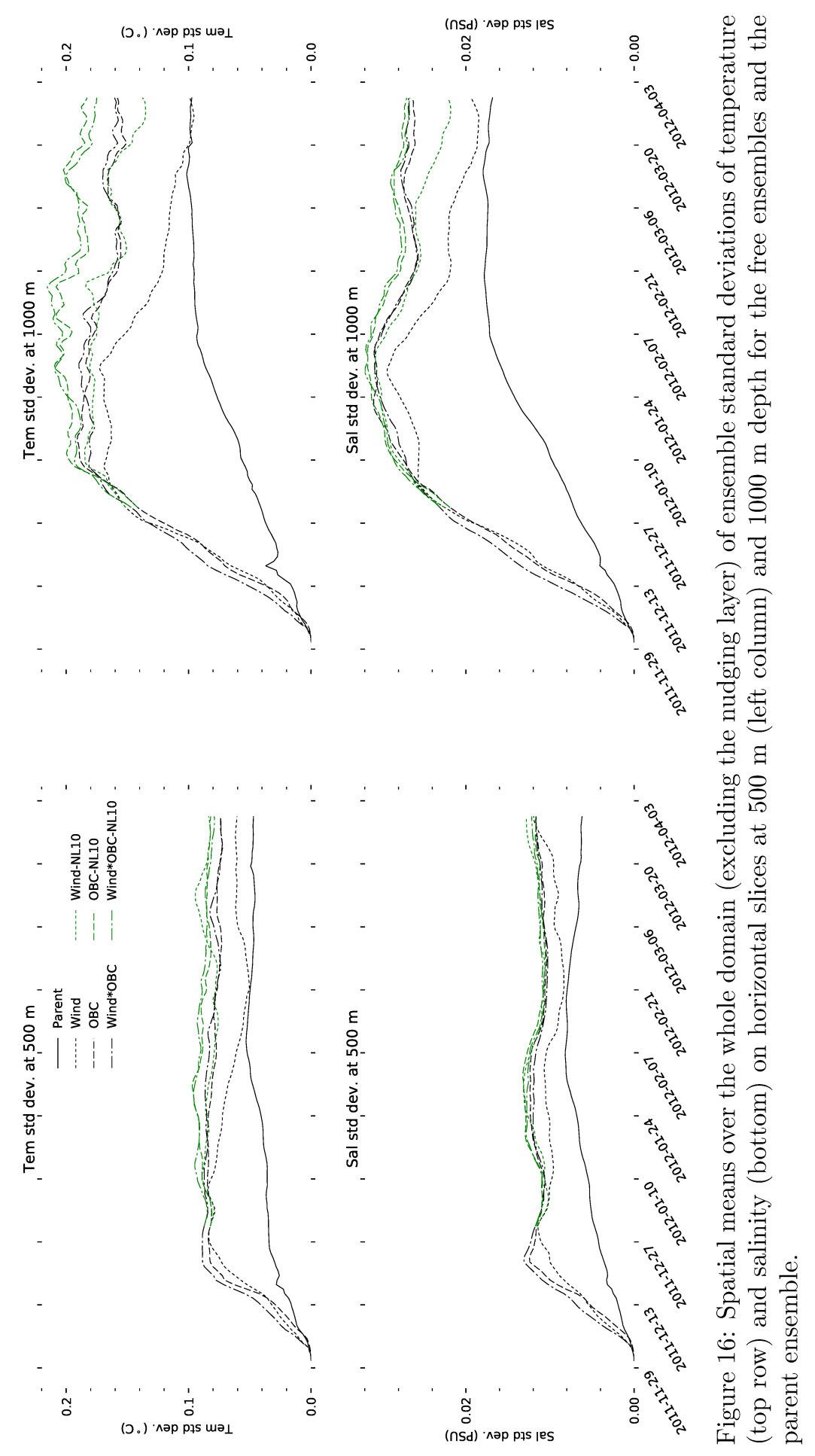



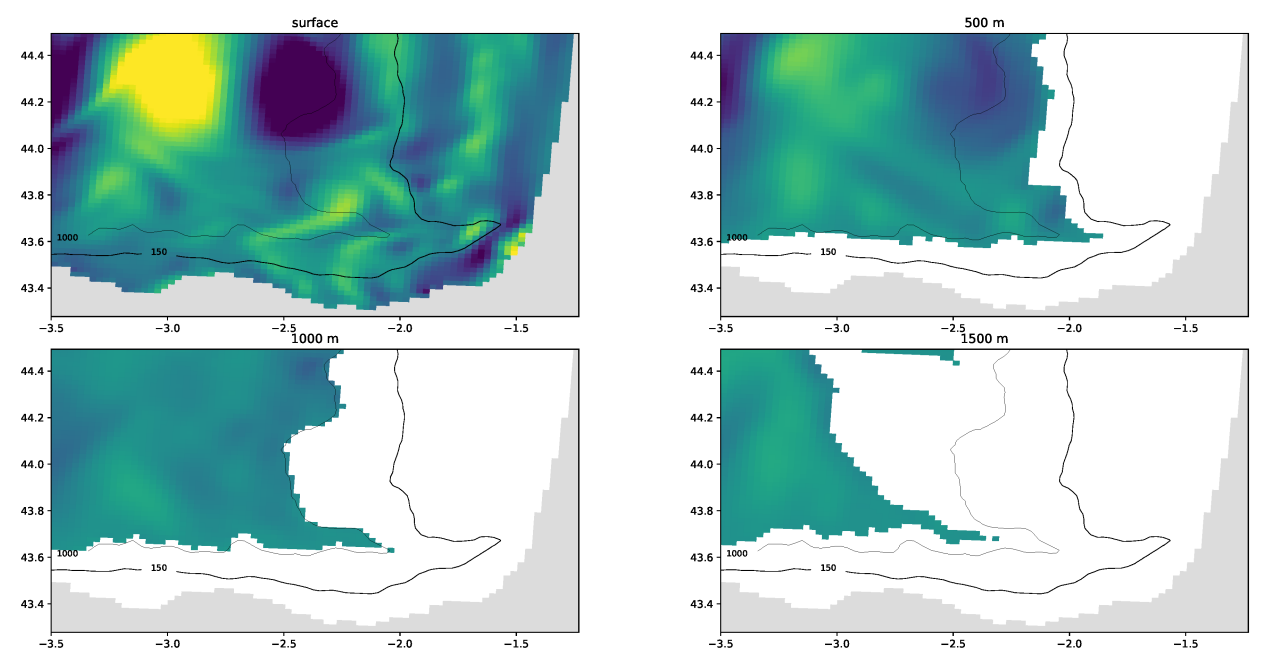

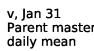

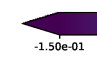

$-1.00-01$

$-5.00 \mathrm{e}-02$
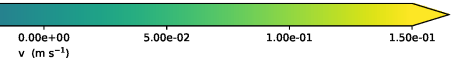

(a)
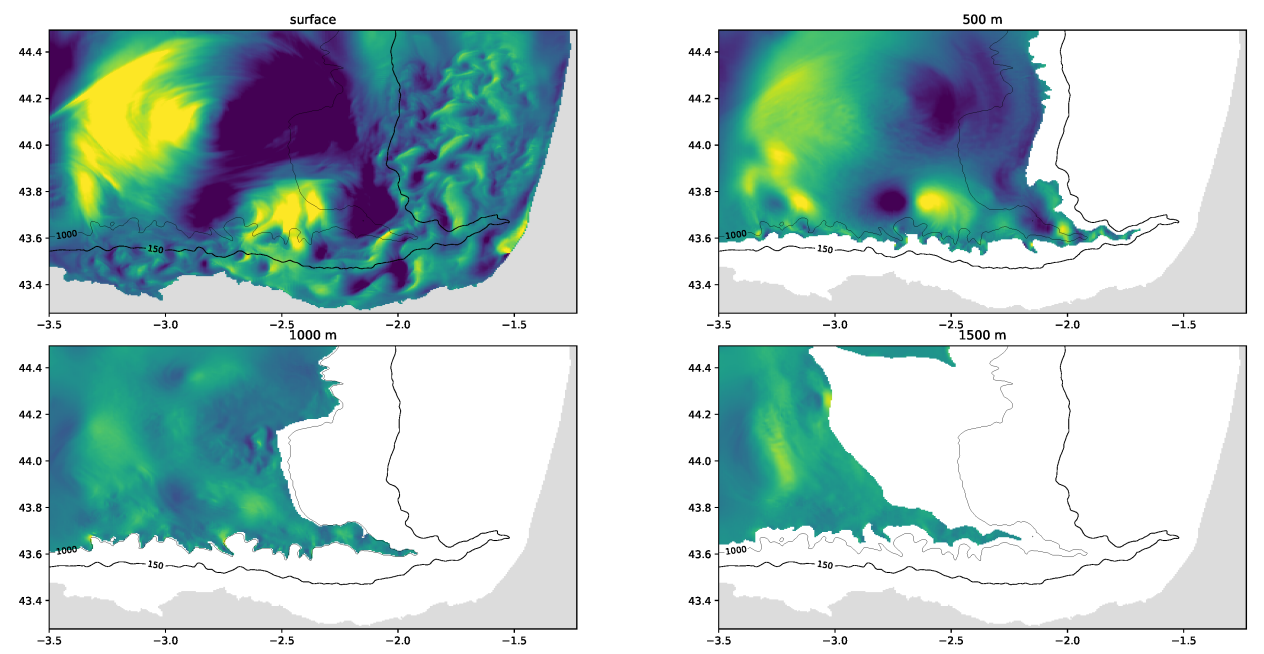

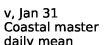

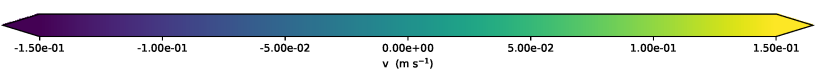

(b)

Figure 17: Meridional velocity at different depths for the unperturbed run of the parent (a) and coastal (b) models. Daily means for 31st January. 


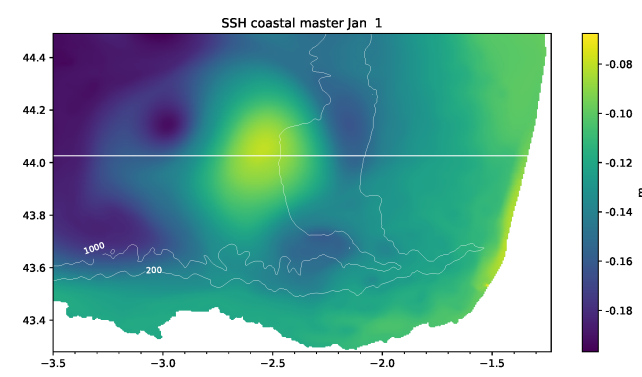

(a)

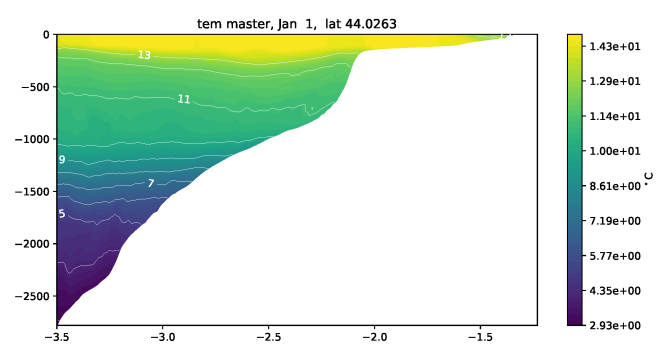

(c)

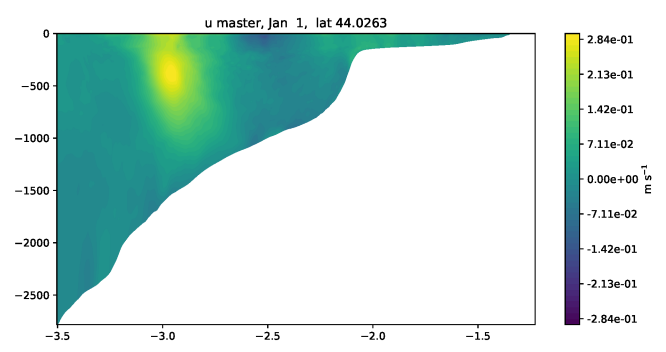

(e)

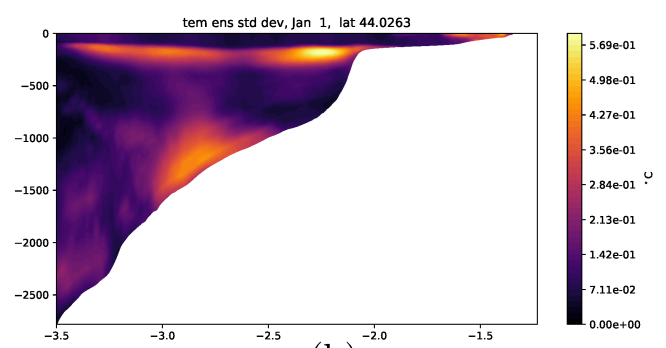

(b)

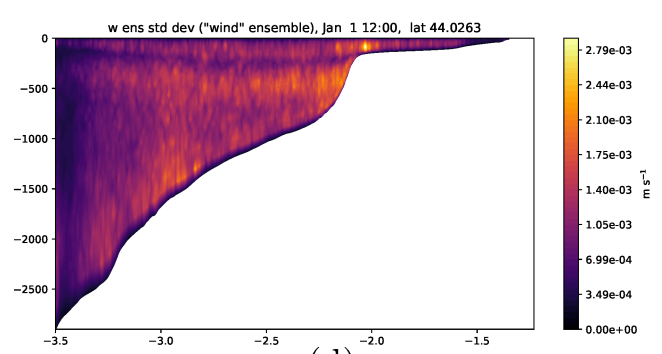

$(\mathrm{d})$

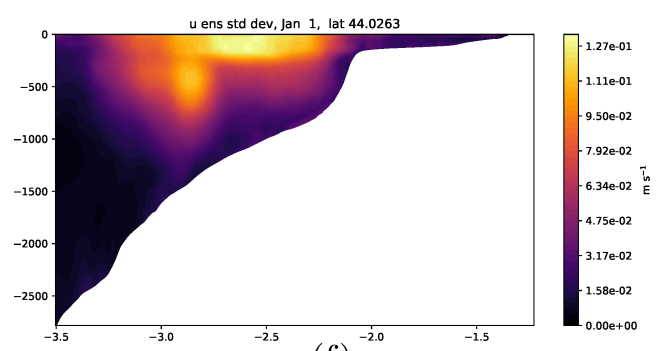

(f)

Figure 18: Illustration of how forcing perturbations at the surface alone can affect the model response at depth through eddy interaction with the bottom-plots for the 1st of January, 2012. In (a) the horizontal line shows where the cross section in subsequent plots is taken, and 200 and $1000 \mathrm{~m}$ depth contours are shown. (a) shows the SSH for the unperturbed model (with the atmospheric inverted barometer effect removed from the SSH signal); (b) the standard deviation of temperature; (c) the temperature for the unperturbed model; (d) is the standard deviation of vertical velocity; (e) the zonal velocity from the unperturbed model; (f) the standard deviation of zonal velocity. The vertical velocity plot is an instantaneous field, whereas the others are daily means. 


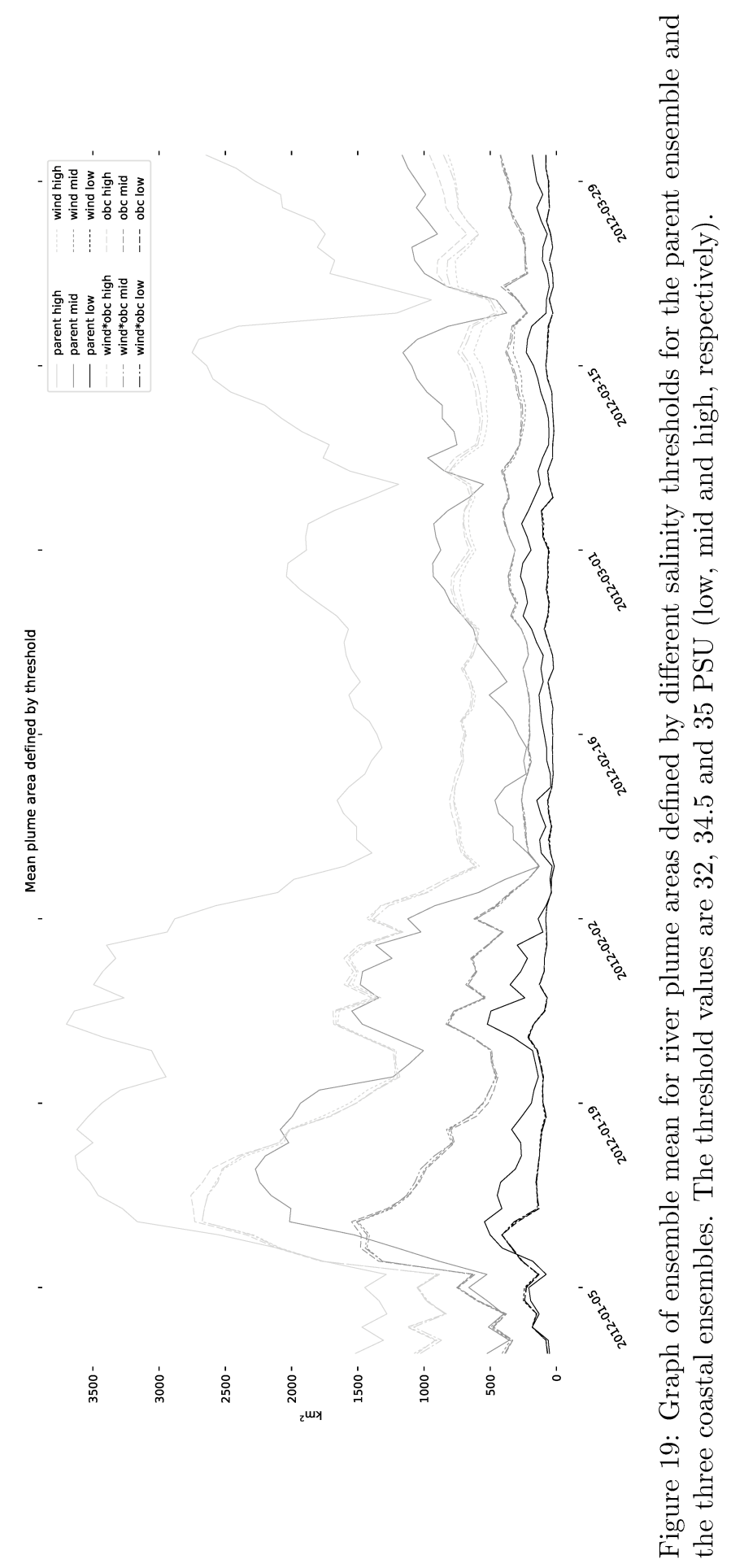




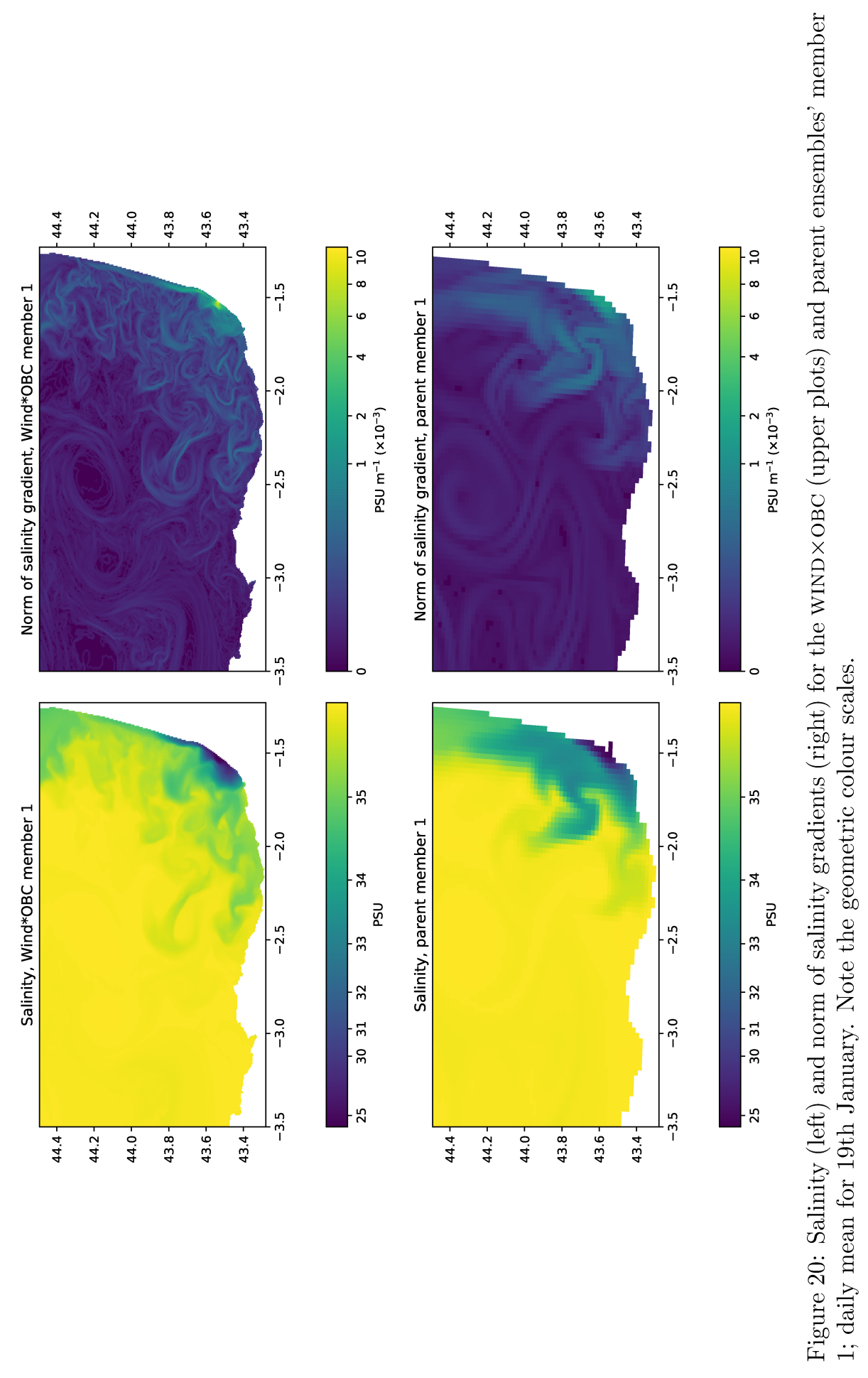

